HANnes KuCH Und StefFEn KitTy HerRmann

\title{
Symbolische Verletzbarkeit und sprachliche Gewalt
}

Gewalt - das ist das Messer, das in den Magen sticht, die Faust, die das Gesicht trifft, der Stein, der den Kopf verletzt. Bei einem Blick auf einschlägige Konzepte der Gewalt wird rasch deutlich: Im Zentrum des Verständnisses von Gewalt steht der machtvolle Zusammenprall zweier Körper, oftmals vermittelt durch körperliche Gegenstände. „Gewalt ist körperlicher Einsatz, ist physisches Verletzen und körperliches Leid [...]«, so der Gewaltsoziologe Trutz von Trotha. ${ }^{1}$ Vorausgesetzt wird mit solch einem Gewaltbegriff mehrerlei: Erstens nämlich, dass das Antun von Gewalt nicht einfach nur in einer Verletzung besteht, sondern in der Verletzung des Körpers eines Menschen. Und zweitens, dass das Mittel, durch das diese Verletzung zugefügt wird, selbst körperlich ist, nämlich ein Teil des menschlichen Körpers oder ein körperlicher Gegenstand. Ein solches Gewaltverständnis legt nahe, dass Sprache keine Gewalt zufügen kann, weil sie lediglich >symbolisch kann verletzen - und das nicht obwohl, sondern gerade weil sie symbolisch ist. Denn sprachliche Gewalt zielt gerade nicht auf die Schädigung der materiellen Existenz einer Person, sondern auf ihr symbolisches Leben. Und in dieser Sphäre, so wird sich zeigen, ist sprachliche Gewalt nicht weniger >real« oder >effektiv als physische Gewalt - auch sie kann in letzter Konsequenz tödlich sein.

Symbolische Gewalt ist auch in den scheinbar nacktesten Gewalttaten immer schon am Werk. Nehmen wir ein Beispiel: Die Folter kann als ein Paradigma absoluter physischer Gewalt gelten. Das Zufügen von möglichst ausgedehnten

1 Trotha, Trutz von, »Zur Soziologie der Gewalt«, in: ders. (Hg.), Soziologie der Gewalt, Sonderheft Kölner Zeitschrift für Soziologie und Sozialpsychologie, Wiesbaden 1997, S. 9-56, hier S. 26. 
Schmerzen in der Folter dient der Intensivierung des Leids. Der Schmerz soll sich in eine Qual ohne Ende verstetigen - wenn nicht real, so doch in der erlebten Zeit des Opfers, die sich in zäher Endlosigkeit dehnt. Doch die Tortur der Folter ist wie kaum eine andere Gewaltform mit dem Symbolischen verschlungen. ${ }^{2}$ Nicht nur das Vorführen der Instrumente, das den Schmerz gleichzeitig androhen und vorwegnehmen soll, sondern auch die Folterung selbst zielt auf die Demonstration von Macht und Überlegenheit. Dem Opfer soll vor Augen geführt werden, dass sein Leben ganz in den Händen eines Anderen liegt, dass es diesem auf Gedeih und Verderb ausgeliefert ist. Es geht darum, das soziale Verhältnis zwischen Folterer und Gefoltertem zur Schau zu stellen. Dasselbe gilt für die Technik des Verhörs, die oft mit der Folter einhergeht: Der monotone, nie endende Rhythmus der Fragen, und vor allem ihre absurde Belanglosigkeit, machen deutlich, dass es im Verhör um vieles geht, vorrangig jedoch nicht um das Gewinnen von Information. Das Opfer soll sagen, was sein Folterer hören will. Damit offenbart das Verhör den tieferliegenden Zweck der Folter: Sie zielt nicht allein darauf ab, den Körper zu quälen, sondern durch die Demonstration von Überund Unterlegenheit ihr Opfer zu demütigen.

Kommen wir von diesem Extrem physischer Gewalt zu ihrer untersten Schwelle: der Ohrfeige. Was heißt es, eine Ohrfeige zu bekommen? In welcher Hinsicht verletzt sie? Natürlich kann die Ohrfeige in einem Vokabular physischer Gewalt beschrieben werden, etwa wenn wir von einer $>$ schallenden Ohrfeige $<$ reden - der Referenzpunkt ist zweifellos der Körper. Doch es liegt auf der Hand, dass sich die verletzende Kraft nicht in dieser körperlichen Dimension erschöpft. Denn die Ohrfeige kann in ihrer physischen Verletzungsmächtigkeit so geringfügig, so wenig intensiv sein, dass sie beinahe belanglos sein müsste. Aber sie ist nicht belanglos. Sie demonstriert etwas: die symbolische Verachtung und Geringschätzung der Akteurin gegenüber der Betroffenen. Auch wenn die Ohrfeige eine körperliche Handlung ist, so zielt sie doch vor allem auf eine symbolische Sphäre: Sie verletzt nicht eigentlich den Körper, sie verletzt das soziale Sein. ${ }^{3}$ Für Levinas stellt die Ohrfeige daher die >exemplarische Szeneく symbolischer Gewalt dar - einer Gewalt, der wir nicht in erster Linie als körperliche, sondern als soziale Wesen ausgesetzt sind. ${ }^{4}$

2 Dazu sehr eindrücklich Scarry, Elaine, Der Körper im Schmerz. Die Chiffren der Verletzlichkeit und die Erfindung der Kultur, Frankfurt / Main 1992, S. 43-90.

3 Manche Autoren gehen soweit zu sagen, dass gerade diese symbolische Dimension, auf die ein Gewaltakt abzielt, eine grundlegende Existenzweise des Menschen freilegt. Für Richard Rorty etwa ist eine Person ein Wesen, das gedemütigt werden kann (Rorty, Richard, Kontingenz, Ironie und Solidarität, Frankfurt / Main 1992, S. 156). Ganz ähnlich formuliert Margalit: »Nur der Mensch leidet unter der Variante der Grausamkeit, die Demütigung heißt und zu der etwa das Nachäffen von Stotterern gehört«. (Margalit, Avishai, Politik der Würde. Über Achtung und Verachtung, Frankfurt / Main 1999, S. 112)

4 Levinas, Emmanuel, Humanismus des anderen Menschen, Hamburg 2005, S. 94. 
Während die Ohrfeige noch ein Mittleres aus physischer und symbolischer Gewalt darstellt, so ist das verletzende Sprechen die Verkörperung symbolischer Gewalt par excellence. Sprachliche Gewalt richtet sich nicht nur gegen die symbolische Dimension der menschlichen Existenz, sondern wird ebenso ausschließlich im Medium des Symbolischen ausgeführt. Sprachliche Gewalt steht damit konträr zu jenem Gewaltverständnis, das Gewalt nur als physische Gewalt denken kann. Im Folgenden möchten wir zwei Seiten sprachlicher Gewalt nachgehen: Zum einen wollen wir zeigen, warum Menschen durch Worte verwundbar sind (Teil I). Unsere These lautet, dass das Verletzende dieser Gewalt in der Verletzung der sozialen Existenz von Menschen besteht. Zum anderen wollen wir zeigen, wie Gewalt durch Sprache ausgeübt wird (Teil II). Die These ist hier, dass sprachliche Gewalt eine soziale Praxis ist, deren Grammatik in den Bedingungen der Äußerung zu suchen ist.

\section{Symbolische Verletzbarkeit}

Aristoteles hat den Menschen als ein sprachliches Wesen bestimmt. Sprache ist für ihn dasjenige Mittel, durch das sich der Bürger in der Polis über Gerechtes und Ungerechtes, Gutes und Schlechtes, Nützliches und Schädliches austauschen und an der Staatsgewalt zu partizipieren vermag. Für die Auseinandersetzung in der Polis entwickelt Aristoteles daher auch seine Rhetorik, die der Wahrheitsfindung und Überzeugung der Adressaten dienen soll. Sprache ist in dieser Sicht das klassische Medium des Diskurses, sie ermöglicht den Austausch von Argument und Gegen-Argument und dient der Übermittlung von Informationen und der Verständigung. Emile Benveniste hat diesen Gedanken so formuliert: »Wir finden in der Welt einen sprechenden Menschen, einen Menschen, der mit einem anderen Menschen spricht, und die Sprache lehrt die Definition des Menschen schlechthin. $\ll^{5}$ Dennoch bleibt auch in dieser Sicht die Sprache den Menschen letztlich äußerlich; sie ist lediglich ein Werkzeug, dessen sie sich bedienen, um mit anderen Menschen in einen Dialog zu treten. Doch Sprache ist nicht nur ein Mittel der Verständigung, das zwischen $>$ Ich $<$ und $>$ Du< vermittelt, sondern auch und vor allem eine Instanz, welche >Ich< und >Du< allererst ins Leben ruft. Dass Menschen sprachliche Wesen sind, bedeutet nicht zunächst, dass sie Sprechen können, sondern dass ihre Existenz aus Sprache gestrickt ist - einer Sprache, die immer vom anderen Menschen her kommt. Genau diese Tatsache aber lässt Menschen in einer immerwährenden Ausgesetztheit gegenüber den Anderen existieren, durch die sie in einem grundlegenden Sinne verletzungsoffen für sprachliche Gewalt sind.

5 Benveniste, Emile, »Über die Subjektivität in der Sprache«, in: ders., Probleme der allgemeinen Sprachwissenschaft, München 1974, S. 287-297, hier S. 288. 


\section{Ansprache und Antwort}

Im Angesprochenwerden gründet die soziale Existenz von Menschen - die Ansprache durch eine Andere führt unvermeidlich ins Soziale ein. Das liegt daran, dass wir in dem Moment, in dem wir angesprochen werden, als ein Jemand adressiert werden. Jede Form der Ansprache - und sei sie noch so kümmerlich zeichnet sich dadurch aus, dass sie sich an Jemanden richtet und mit Jemandem kommuniziert. Selbst eine achtungslose Aussage wie »Du Niemand« bestätigt noch kommunikativ, was sie semantisch negiert. Obwohl sie versichert, dass die Adressatin ein Niemand sei, ist es doch ein Jemand, dem sie mitteilt, Niemand zu sein. Jede Ansprache ist daher ein Akt der Anerkennung, wobei diese Anerkennung noch gar nicht in einem normativen Register der Wertschätzung gelesen werden kann, sondern dafür steht, dass sich jede Anrede an eine Adressatin wendet. Es sind daher nicht erst die sprachlichen Bekundungen von Lob, Wohlwollen oder Befürwortung, die Anerkennung ausdrücken, sondern viel grundlegender ist jede Ansprache in einer bestimmten Hinsicht schon ein Ausdruck der Anerkennung. Anders ausgedrückt: Jeder Ansprache ist unabhängig von ihrem konkreten semantischen Gehalt eine grundlegende Form der Anerkennung eigen. Und diese Anerkennung, so schreibt Judtih Butler im Anschluss an Louis Althusser, »wird zu einem Akt der Konstitution «, weil sie die Angesprochene im Moment der Ansprache als soziales Wesen anerkennt. ${ }^{6}$ Auch wenn die Ansprache behauptet, ihre Adressatin sei nur ein Ding oder ein Tier, wendet sie sich doch an Jemanden, dem sie sagt, er sei ein Ding oder ein Tier - und nicht an ein Ding oder ein Tier. ${ }^{7}$ Wer angesprochen wird, wird als Mensch unter Menschen adressiert, als Eine unter Anderen, als Jemand im Kreis von Vielen - damit begründet die Ansprache in fundamentaler Weise das soziale Sein.

Der Ansprache können wir uns nicht entziehen, denn jede Ansprache eröffnet einen Raum der Kommunikation. In dem Moment, wo wir durch ein persönliches »Du«, ein höfliches »Sie« oder ein schroffes »Hey« adressiert worden sind, sind wir der Ansprache in grundlegender Weise ausgeliefert. Jede Haltung, die wir als Angesprochene einnehmen, ist unweigerlich kommunikativ. Angesprochen zu werden bedeutet, dass mein Verhalten stets mitteilsam sein wird: Unabhängig davon, auf welche Weise genau ich mich verhalte, und unabhängig davon, ob ich will oder nicht, die Ansprache zieht mich in den Sog der Kommunikativität, in dem es nicht möglich ist, nicht zu kommunizieren. ${ }^{8}$ Wir sind in eine Beziehung zum Anderen gestellt, der wir uns nicht entziehen können.

6 Butler, Judith, Hass spricht. Zur Politik des Performativen, Berlin 1998, S. 43. Und an anderer Stelle: »Angesprochen werden bedeutet also nicht nur, in dem was man bereits ist, anerkannt zu werden, sondern jene Bezeichnung zu erhalten, durch die die Anerkennung der Existenz möglich wird.« (Ebd., S. 15)

7 Vgl. dazu auch Margalit, Politik der Würde, a.a.O., S. 114-142.

8 Watzlawik, Paul / Janet H. Beavin / Don D. Jackson, Menschliche Kommunikation. Formen, Störungen, Paradoxien, 10. Aufl., Bern u.a. 2000, S. 50 ff. 
Die Haltung, die auf die Ansprache folgt, kann grundlegend zwei Formen annehmen: Die Ansprache kann, wie wir mit Levinas sagen können, zur >Verantwortung r rufen. ${ }^{9}$ Das heißt, sie fordert dazu auf, die Möglichkeit zu nutzen, die jedem Angesprochenwerden innewohnt: das Antworten. Die Ansprache kann so zum Gespräch werden, durch das wir das soziale Band zur Anderen aufgreifen und weiter knüpfen können. ${ }^{10}$ Die Antwort, die wir auf die Ansprache geben, kann ganz unterschiedlich aussehen. Sie kann die Andere grüßen, sie zurechtweisen, sie abweisen, kurz: Sie kann ihr mehr oder weniger gerecht werden. In jedem Fall nimmt sie die >Ver-Antwort-ung « wahr, der Ansprache durch die Andere zu antworten und diese dadurch selbst zu einer Angesprochenen zu machen.

Die Haltung, die durch die Ansprache hervorgerufen wird, kann jedoch auch noch eine andere Form annehmen: das Schweigen. Auch wenn das Schweigen immer noch kommunikativ ist, so stellt es doch das Ausbleiben einer Antwort dar: Seine Stummheit spricht nicht zurück. Es verweigert eine Antwort. Anders gesagt: Man kann zwar nicht nicht kommunizieren, aber man kann das Sprechen beenden und zu schweigen beginnen - und damit in einem grundlegenden Sinn auf die Gabe der Antwort verzichten. Die Möglichkeit des Schweigens hat zur Folge, dass die Einführung ins Soziale nur aus der passiven Perspektive des Angesprochenwerdens gesichert werden kann, nicht jedoch aus der aktiven Perspektive des Sprechens. Denn die Sprecherin, die in ihrer Ansprache behauptet, sie sei ein soziales Wesen, vielleicht sogar ein wichtiges, muss darauf keine Antwort bekommen. Die Ansprache hat sich in diesem Fall zwar an jemanden gerichtet, doch da sie keine Antwort erhält, wird sie nicht als ein Sprechen anerkannt, das von jemandem kommt. Sprechen ist der Appell an eine Antwort, doch die Antwort kann immer auch ausbleiben. ${ }^{11}$ Das Sprechen bleibt dann bloßes Gebrabbel, Gemurmel oder Störgeräusch. Anders gesagt: Wir können uns nicht selbst ins Soziale sprechen, ins Soziale werden wir immer nur durch Andere gesprochen. Diese Abhängigkeit von der Ansprache begründet das Ich in einer fundamentalen Passivität - auch dort, wo es ihr in der Aktivität des Sprechens zu entkommen sucht. Auch der selbstinitiierten Rede des Subjekts liegt das Streben nach einem Angesprochenwerden zugrunde: ich spreche, um eine Antwort zu bekommen. Selbst dort, wo ich derjenige bin, der redet, geht es mir immer auch darum, selbst angesprochen zu werden. Doch wer spricht, muss nicht unbedingt auch angesprochen werden. Dort, wo das Ich am aktivsten scheint, kommt es folglich - gleichsam hinter seinem Rücken - zu einer Radikalisierung seiner Passivität.

9 Levinas, Emmanuel, Ethik und Unendliches. Gespräche mit Philippe Nemo, hg. v. Peter Engelmann, Wien 1996.

10 Vgl. dazu etwa Buber, Martin, Das dialogische Prinzip, Heidelberg 1984.

11 Zum Appellcharakter des Sprechens vgl. Lacan, Jacques, »Funktion und Feld des Sprechens und der Sprache in der Psychoanalyse«, in: ders., Schriften I, Olten 1973 S. 71-169, hier S. 84 ff. 


\section{Sagen und Gesagtes}

Ansprache und Antwort fördern eine Dimension der Sprache zutage, in der wir nicht nur oder nicht in erster Linie sprechen, um Aussagen über die Welt zu machen oder um einem >Empfänger〈Informationen zu übermitteln. Ein semantisches Verständnis jedoch betrachtet Sprache vor allem im Hinblick auf die Möglichkeit, mit ihrer Hilfe konstatierende Äußerungen über eine ihr entgegensetzte Welt zu machen. Bestimmte Formen von Äußerungen, nämlich Aussage-Sätze bzw. konstatierende Äußerungen, also Äußerungen, die Tatsachen feststellen, sind in diesem Bild der Sprache wahrheitsfähig, das heißt, sie können wahr oder falsch sein. Viele Kommunikationstheorien wiederum verstehen Sprache und Sprechen nach einem Modell, das bei der Intention des `Senders`, etwas mitzuteilen, beginnt, zur Übermittlung des `Gehalts` der Mitteilung durch Zeichen voranschreitet, zur Wahrnehmung des Zeichens durch einen `Empfänger`gelangt, um schließlich in der >Decodierung ১ durch den Empfänger zu enden. Ansprache und Antwort zeigen jedoch eine Modalität des Sprechens, in der es weder darum geht, Aussagen über die Welt zu machen, noch Informationen zwischen Ich und Anderer auszutauschen, sondern vielmehr um das Verhältnis von Selbst und Anderer. Im Sprechen geht es immer auch um die bloße Eröffnung einer Beziehung zur Anderen, noch vor jeder Semantizität des Ausgesagten. Sprechen ist nie nur die zweckgerichtete Mitteilung von Information, vielmehr reden wir in bestimmtem $\mathrm{Ma} \beta$ auch deshalb, um die Andere im Sprechen anzuerkennen und selbst anerkannt zu werden. Wir sprechen, weil Worte gewissermaßen der Stoff sind, aus dem wir gemacht sind. Mit Levinas nennen wir diese Dimension der Sprache das >Sagen $<$ und unterscheiden sie vom >Gesagten $<.{ }^{12}$ Das Sagen meint dabei keineswegs die aktive Form des Gesagten, so, als wäre das Gesagte das Produkt der Aktivität der Praxis des Sagens. Das Sagen bezeichnet vielmehr eine Dimension der Sprache, in der das an die Andere gerichtete Wort immer »die symbolische Gabe eines Sprechens $\aleph^{13}$ ist. Während im Gesagten Bedeutung übermittelt wird oder Ansprüche auf Wahrheit erhoben werden, wird im Sagen zunächst allein eine Nähe zwischen den Sprechenden eröffnet. Im Sagen geht es folglich um das Faktum des Kommunizierens, nicht um das Kommunizierte: Im Sagen zählt nicht das >Was〈, sondern das >Dass $<$ des Sprechens. ${ }^{14}$ Was kommunikationstheoretisch als Redundanz in der Sprache betrachtet wird, bildet im Horizont des Sagens einen Resonanzraum für die Andere. Nicht die Übermittlung von Information, sondern die Evokation einer Antwort steht im Vordergrund des Sagens.

Das Sagen als intersubjektive Dimension der Sprache zeigt sich in manchen sprachlichen Handlungen besonders deutlich, vor allem dort, wo Sprache de-

12 Levinas, Emmanuel, Jenseits des Seins oder anders als Sein geschieht, Freiburg, München 1998, S. 110 ff.

13 Lacan, »Funktion und Feld des Sprechens«, a.a.O., hier S. 133.

14 Diese Formulierung verdanken wir: Till, Sabine, Sprache und Stimme bei Lacan und Levinas, Magistraarbeit, Institut für Philosophie, Freie Universität Berlin 2005, S. $12 \mathrm{ff}$. 
semantisiert auftritt. Begrüßungsformeln wie das $\gg \mathrm{Na}$, wie geht's?〈, der kurze Small-Talk zwischen Tür und Angel, mehr oder weniger formelle Erkundigungen nach der Gesundheit, die Feststellung von selbstverständlichen Sachverhalten oder Bemerkungen über das Wetter - all diese Äußerungen zeichnen sich dadurch aus, dass sie nicht (oder zumindest kaum noch) darauf abzielen, dem Anderen etwas mitzuteilen oder etwas über die Welt auszusagen, sondern nur darauf, eine Beziehung oder eine Nähe zur Anderen zu eröffnen oder das Gespräch mit ihr nicht zu beenden. Auch das rhythmische $>\mathrm{Mhm}<$, das die Rede der Anderen begleitet, ist nie nur eine konstatierende Bestätigung der Äußerungen des Gegenübers, sondern bezweckt immer auch die Fortdauer des Gespräches selbst. Malinowski bezeichnete diese Dimension des Sprechens als sphatische Kommunikation $\prec .{ }^{15}$ Den Begriff der phatischen Kommunikation fand Malinowski bei seinen Studien auf den Trobriand-Inseln im pazifischen Melanesien. Für die dortigen Gemeinschaften spielte das Geben und Empfangen von sprachlichen Gaben in der phatischen Kommunikation eine ähnlich bedeutsame Rolle wie der Austausch von materiellen Gaben - beide Praktiken dienen der Stiftung von Gemeinschaft zwischen den Beteiligten.

Aber selbstverständlich ist es so, dass es im Sprechen, auch wenn wir immer >zu jemandem`sprechen, zumeist doch >um etwas geht. Unser Sprechen hat meist ein Thema und ist somit Gesagtes. Auch Levinas betont, dass das Sagen so sehr im Gesagten aufgeht, dass es in Vergessenheit zu geraten droht. Aber selbst wenn es >um etwas geht, selbst wenn wir nur Informationen austauschen oder eine Feststellung treffen, ist die Äußerung stets auch noch eine symbolische Gabe an den Anderen. Levinas etwa betont, dass sich das Sagen selbst noch in konstatierenden Äußerungen zeigt: Weil der Aussage-Satz (frz. proposition) immer auch ein Angebot (frz. ebenfalls proposition) an den Anderen ist, stellt er eine Modalität der Annäherung an den Anderen dar. ${ }^{16}$

Und mehr noch: Das Sagen zeigt sich meist nicht nur im Gesagten, das Sagen ist sogar zum großen Teil davon abhängig, dass es einen gewissen Bezug zum Gesagten bewahrt. Wir treffen an diesem Punkt also auf ein Spannungsverhältnis zwischen Sagen und Gesagtem: Wenn das Gesagte bedeutungslos wird, droht dem Sagen dasselbe Schicksal - das Sprechen wird zu einem beredten Schweigen. Wenn das $>\mathrm{Mhm}$ ^ meines Gegenübers in unserem Gespräch seinen Rhythmus verliert und mechanisch wird, verlieren seine Äußerungen nicht nur ihre Rolle als Bestätigungen dessen, was ich gesagt habe, sie verlieren auch ihre phatische Dimension, auf die Fortdauer des Gesprächs selbst hinzuwirken. Ein Beispiel dafür finden wir bei Watzlawik, der das Beispiel eines Lobes nennt, das zu jeder Gelegenheit wiederholt wird. ${ }^{17}$ Sofern es nie mit Kritik kontrastiert wird,

15 Malinowski, Bronislaw, »Das Problem der Bedeutung in primitiven Sprachen «, in: Charles K. Ogden / Ivor A. Richards, Die Bedeutung der Bedeutung, Frankfurt / Main 1974, S. 323-384 (engl. Original 1923), hier besonders S. 347 ff.

16 Levinas, Jenseits des Seins, a.a.O., S. 113.

17 Watzlawik / Beavin / Jackson, Menschliche Kommunikation, a.a.O., S. 86 f. 
verliert es nach und nach seine Bedeutung. Dabei verkehrt es sich jedoch nicht in sein Gegenteil, nämlich in Kritik oder Tadel, sondern es wird >leer «. Es gibt dem Anderen zu verstehen, snicht der Rede wert, inexistent zu sein. Indem das Gesagte seine Bedeutung verliert, droht aus dem Sagen ein Schweigen zu werden. Der Abbruch des Sagens kann folglich zu einem >mitteilsamen< Schweigen werden, ein Schweigen, das noch eiserner sein kann als ein buchstäbliches Schweigen.

\section{Name und Benennung}

Bisher ging es darum, dass wir durch die Ansprache als Jemand anerkannt werden und dass sich viele Gesprächsakte unserer alltäglichen Kommunikation gerade auf der Ebene des Sagens befinden. Nun gilt es vom Sagen zum Gesagten fortzuschreiten, um zu sehen, dass wir im Reden nicht nur als Irgend-Jemand, sondern als ein ganz bestimmter So-Jemand anerkannt werden. Anders gesagt: Es geht darum, wie wir durch die Sprache zu einem einzigartigen sozialen Wesen werden.

Die Verleihung des Eigennamens kann als \Urszene< der sozialen Inauguration von Menschen gelten, denn die soziale Existenz, die wir in der Ansprache zugesprochen bekommen, wird im Eigennamen verzeitlicht und dauerhaft gemacht. ${ }^{18}$ Das wird an dem grundlegenden Unterschied zwischen der Ansprache mit einem $>$ Du< und der Ansprache mit dem Eigennamen deutlich: Jeder Gebrauch des Eigennamens bezieht sich auf etwas, das hinsichtlich der verschiedenen Verwendungssituationen immer identisch bleibt. Jede Ansprache mit einem Eigennamen nimmt Bezug auf ein einziges menschliches Wesen. Die Ansprache mit einem >Du< dagegen hat keine kontinuierliche Referenz: >Du< hat seine Kontinuität nur für die Dauer der Ansprache, denn in dem Moment, in dem eine andere Adressatin angesprochen wird, ist $>\mathrm{Du}<$ jemand anderes. Während die Zeit des Eigennamens also ewig ist, ist die Zeit des Pronomens dem Augenblick verhaftet. 〉Du<, so lässt sich mit Benveniste sagen, ist ein >mobiles Zeichen<, dessen Referenz jeweils mit dem Vollzug des Sprechens zustande kommt, der Eigenname hingegen ist ein >starres Zeichen<, dem seine Referenz anhaftet. ${ }^{19}$ Das hat zur Folge, dass der Eigenname die Existenz eines Du auch in dessen Abwesen-

18 Vgl. dazu Butlers Überlegungen zur Anrufung mit dem Eigennamen, die sie im Anschluss an Louis Althusser anstellt (Butler, Hass spricht, a.a.O., S. 41-47, und dies., Psyche der Macht. Das Subjekt der Unterwerfung, Frankfurt / Main 2001, S. 101124, sowie Althusser, Louis, »Ideologie und ideologische Staatsapparate«, in: ders., Ideologie und ideologische Staatsapparate. Aufsätze zur marxistischen Theorie, Hamburg 1977, S. 108-153, hier S. 130-153).

19 Vgl. Benveniste, Emile, »Die Natur der Pronomen«, in: ders., Probleme der allgemeinen Sprachwissenschaft, München 1974, S. 279-286, hier S. 284. Der Begriff >starres Zeichen ausdrücke< [rigid designators] an. Vgl. Kripke, Saul A., Name und Notwendigkeit, Frankfurt / Main 1981, S. 59 f. 
heit verbürgt. Er gewährt Sicherheit, denn seine Nennung bewirkt, dass jemand adressiert ist, unabhängig davon wo er ist, wer er ist oder wie er ist. Um durch den Eigennamen benannt zu werden, bedarf es also keiner besonderen Leistung der Adressatin, sie muss nicht in den Namen hineinwachsen und ihn ausfüllen, er nennt sie einfach: jederzeit. Der Eigenname garantiert, dass $>$ Ich $<$ gestern dieselbe war, die $>I$ ch $<$ heute bin und $>I c h<$ morgen sein werde.

Der Eigenname identifiziert nicht nur ein Leben lang, sondern er versichert seiner Trägerin auch, ein in das Soziale eingeschriebene Wesen zu sein. Das wird an einer Äußerung deutlich, die Wittgenstein ein Leben lang fasziniert hat: >Ich, Ludwig Wittgenstein ${ }^{20}$ Auf den ersten Blick könnte man meinen, es handelt sich dabei um eine Tautologie. Doch ganz im Gegenteil, an diesen Worten lässt sich etwas Grundlegendes zeigen: Im Gegensatz zum indexikalischen Demonstrativpronomen >Ich< haftet dem Eigennamen >Ludwig Wittgenstein ziale Dimension an. Der Name wird uns durch Andere gegeben. Sich beim Namen zu nennen, bedeutet daher, sich aus der Perspektive der Anderen auf sich zurückzuwenden - man könnte fast sagen: sich als einen Anderen anzusprechen, und das heißt: sich auf sich selbst als ein soziales Wesen zu beziehen. Während das Wittgensteinsche >Ich< lediglich ein singuläres, autonomes Wesen nennt, nennt der Eigennamen dieses >Ich< als ein >Ich< unter Anderen: als Teil einer Gemeinschaft. Der Eigenname bietet seiner Trägerin auf diese Weise die Möglichkeit, sich durch die Augen einer Anderen hindurch auf sich >selbst $<$ zu beziehen: Das >Ichく wird dadurch zum >Selbst.

Der Eigenname ist zugleich die konkreteste und die abstrakteste Form der Selbstheit. Konkret ist der Eigenname, weil er unumstößlich die Existenz nennt und damit einen Ort eröffnet, an dem jemand adressiert werden kann. Abstrakt bleibt er jedoch, weil er außer dieser Existenzaussage keinerlei weitere Aussage macht. Aufgrund seiner kompletten Indifferenz gegenüber jeglicher Bedeutung gibt er keinerlei Auskunft über seine Trägerin. Das Selbst droht so, ein IrgendJemand zu bleiben - oder um Paul Ricœur zu paraphrasieren: der Eigenname droht das Selbst auf Selbigkeit festzuschreiben. ${ }^{21}$ Um das zu verhindern und das Selbst als ganz bestimmten So-Jemand im Sozialen zu situieren, bedarf es gesättigter Formen der Ansprache. Dazu gehören etwa gesellschaftliche Namen, Klassifizierungen, Prädikate und Beschreibungen, die wir an dieser Stelle unter dem Begriff der >Benennung zusammenfassen wollen. ${ }^{22}$

20 Vgl. exemplarisch Wittgenstein, Ludwig, Das blaue Buch, Frankfurt / Main, 9. Aufl., 2000, S. 107.

21 Ricœur, Paul, Das Selbst als ein Anderer, München 1996, S. 39-54.

22 Eine ähnliche Unterscheidung findet sich auch bei Butler, die zwischen >Eigen-

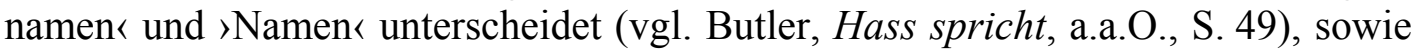
bei Bourdieu, der >Eigennamen` und `Gattungsnamen ‘ differenziert (Bourdieu, Was heißt sprechen? Zur Ökonomie des sprachlichen Tausches, 2., erw. und überarb. Aufl., Wien 2005, S. 99). 
Diejenige Benennung, die dem Eigennamen noch am nächsten ist, aber doch schon in einem ganz anderen Register stattfindet, ist die Benennung mit einem Schimpf- oder Kosenamen. Diesen geht es nicht nur darum, ihr Gegenüber anzusprechen, sondern beide wollen auch etwas über ihren Adressaten aussagen: Das abwertende `Schwitze-Sven< hebt genauso wie das liebevolle `Moos-Roseく eine Eigenschaft des Adressaten vor, es ist mit Gehalt gesättigt, in ihnen kann sich die adressierte Person als ein so-und-so bestimmter Jemand wiederfinden. Dieser kann sich selbst als ganz bestimmtes soziales Wesen unter anderen sozialen Wesen verorten: als So-Jemand, der So-Jemandem gegenübertritt. Wird beispielsweise eine Schülerin von ihrem Lehrer als >faul bezeichnet, dann haben wir es mit einer Benennung zu tun, welche die Angesprochene im Bezug auf ihre Tüchtigkeit nicht als >fleißig`oder >arbeitsam`, sondern als >faul klassifiziert. Dadurch erhält die Schülerin einen genau bestimmten Platz, von dem aus sich ihr die soziale Welt auftut und von dem aus sie anderen gegenüber Stand gewinnen kann: Sie kann sich etwa den Freunden gegenüber mit ihrer lässigen Art brüsten, muss sich den Eltern gegenüber wahrscheinlich rechtfertigen und von den Lehrern weiterhin Tadel einstecken. Kurz: Sie wird So-Jemand, denn die Benennung gibt ihr die Möglichkeit, einen sozialen Ort einzunehmen, der nicht existieren würde, wäre sie nicht adressiert worden. Die Benennung versetzt an einen sozialen Ort und in eine geschichtliche Zeit, sie schafft, wie Judith Butler schreibt, »die sprachliche Gelegenheit des Individuums, Verständlichkeit zu gewinnen und zu reproduzieren, also die sprachliche Bedingung seiner Existenz und Handlungsfähigkeit. $\ll^{23}$

Je konkreter die Benennungen sind, die wir erfahren, desto mehr sind sie auch einem bestimmten Kontext verhaftet und verfallen mit der Zeit. Das mag daran liegen, dass wir uns entwickeln und verändern, oder aber einfach daran, dass wir in verschiedenen Kontexten ganz unterschiedlich adressiert werden. Wir haben es mit einer Asynchronizität zwischen Benennung und Eigennamen zu tun: Der Eigenname ist ewig, tendiert jedoch dazu, das Selbst als Irgend-Jemand festzulegen, Benennungen dagegen entfalten das Selbst als So-Jemand, sind dafür aber vergänglich. Die Stiftung des Selbst als So-Jemand steht damit immer in einem Spannungsverhältnis zur Vergänglichkeit der Benennung, denn zu So-Jemandem werden wir nie durch einen einzigen, singulären Akt, wie es bei der Verleihung des Eigennamens der Fall ist, sondern nur durch fortwährende Akte der Benennung. Um So-Jemand zu sein, müssen wir immer wieder von Neuem benannt werden. Diese fortwährende Benennbarkeit führt dazu, dass wir für die Namen und Ausdrücke, mit denen wir gerufen werden, eine grundlegende Offenheit besitzen.

23 Butler, Psyche der Macht, a.a.O., S. 15. 


\section{Abhängigkeit und Anerkennung}

Im Gegensatz zum Eigennamen, der uns einfach verliehen wurde, stellt sich bei anderen Formen der Benennung die Frage, warum wir sie akzeptieren sollten. Können sie nicht auch einfach abgelehnt werden? Noch dazu, wenn es sich um eine negative Benennung handelt? Diese Fragestellung findet sich auch in einer Geschichte verdichtet, die von einem jüdischen Mädchen handelt, das zum Ende des Nationalsozialismus aus einem Konzentrationslager befreit wird. Nach der Befreiung wird es von den alliierten Soldaten nach seinem Namen gefragt. Seine Antwort lautet: »Judensau«. Diese Antwort macht deutlich, dass wir manchmal verletzende Benennungen akzeptieren. Denn obwohl jede Ansprache immer auch die Möglichkeit einer ablehnenden Antwort birgt, kann diese doch den Preis haben, zukünftig vielleicht gar keine Ansprache mehr zu erfahren. Manchmal nehmen wir daher lieber in Kauf, eine verletzende Ansprache anzunehmen, bevor wir unsichtbar bleiben. Das macht deutlich, dass wir von der Ansprache durch Andere in so grundlegendem Maße abhängig sind, dass wir auch verletzende Ansprachen akzeptieren. Dieser Umstand ist in der Zeitlichkeit jeder Ansprache verwurzelt: Der soziale Ort, der uns durch Benennungen zugewiesen wird, ist nie abschließend besetzt. Die soziale Existenz, welche die Ansprache gewährt, ist immer unabgeschlossen, sie muss durch fortwährenden Akte der Ansprache immer wieder erneuert und aufrechterhalten werden. Weil wir über die Zeit hinweg immer wieder neuen Benennungen unterliegen können und weil Benennungen in Vergessenheit geraten oder einfach nicht weiter überliefert werden können, tendieren sie immer wieder dahin zu verblassen und müssen daher durch andere, neue Benennungen ersetzt werden. Durch diese begrenzte Zeitlichkeit der Benennung stehen wir in einer dauerhaften Abhängigkeit von der Ansprache durch andere. Als soziale Wesen sind wir andern Menschen ausgesetzt, liegt unsere Existenz in ihrer Hand, sind wir - um eine Levinas'sche Redewendung aufzugreifen - die >Geisel der Anderen «. In dieser Ausgesetztheit gründet die symbolische Verletzungsoffenheit eines jeden Menschen. Sie macht uns in einem grundlegenden Sinne empfänglich für jede Form der verletzenden Ansprache. Sprachliche Verletzbarkeit tritt also nicht einfach zu den sozialen Beziehungen, die Menschen unterhalten, hinzu, sondern sie ist, wie Judith Butler treffend formuliert, »eine der ursprünglichen Formen, die diese sozialen Beziehungen annehmen «. ${ }^{24}$ Das Verhältnis von Selbst und Anderer ist also ein fundamental asymmetrisches. Weder in der Ausgangsstruktur noch in einem auch nur möglichen zukünftigen Horizont befinden sich Selbst und Andere in einem Verhältnis der Gleichheit, Symmetrie oder Wechselseitigkeit. Die symbolische Verletzung zerstört folglich keine vorgängige Gleichheit oder Symmetrie, sondern beutet eine primäre Asymmetrie zwischen Selbst und Anderer aus. 
Dass Intersubjektivität grundlegend symmetrisch zu denken sei, ist ein Grundaxiom der universalpragmatischen Kommunikationstheorie. ${ }^{25}$ Ein prominenter Ausgangspunkt dieses Denkens ist die Hegelsche Herr-Knecht-Dialektik. Der `Kampf um Anerkennung`, den die beiden Selbstbewusstseine hier austragen, läuft darauf hinaus, dass Anerkennung nur wechselseitig und unter Gleichen möglich ist - gerade weil die Anerkennungsbeziehung zwischen Herr und Knecht scheitert: Der Herr will von seinem Gegenüber seine Wesentlichkeit bestätigt bekommen, und zugleich zielt er darauf ab, seinem Gegenüber keine Wesentlichkeit zuzugestehen. Damit steuert er in eine »existentielle Sackgasse ${ }^{26}$ : Denn wie soll sich der Herr anerkannt fühlen, wenn er denjenigen, von dem er anerkannt wird, nicht selbst anerkennt? Was zählt die Anerkennung von jemandem, der nichts zählt? Sie ist schlichtweg wertlos. Hegel sieht in diesem Widerspruch einen Grund für das Scheitern der Herrschaft des Herrn. Daher wird in einer hegelianischen Denktradition die Szene der Anerkennung zumeist so verstanden, dass sie mit einem gleichsam zwingenden Telos auf eine Symmetrie zusteuert, in der sich Herr und Knecht wechselseitig anerkennen. ${ }^{27}$

Das Grundproblem dieser Position besteht jedoch darin, dass sie nie über eine dyadische Relation hinausdenkt: Ein Selbstbewusstsein trifft auf ein anderes; der Dritte kommt in diesem Narrativ nicht vor - obwohl eine triadische Struktur die Dynamik des Kampfes auf grundlegende Weise verändert. ${ }^{28}$ Mit dem Auftauchen eines Dritten neben Herr und Knecht wird aus der interpersonalen Beziehung eine gesellschaftliche Szene, womit ein struktureller Bruch von einer wechselseitigen hin zu einer machtgesättigten Dynamik markiert ist. Denn die Bewegung auf Reziprozität hin wird dadurch gebrochen, dass der Herr seine Anerkennung nun auch von einer dritten Person erhalten kann - und diese Anerkennung erfolgt nicht nur trotz, sondern manchmal vielleicht sogar gerade aufgrund des Umstandes, dass es mit dem Knecht jemanden gibt, der ihm unterlegen ist. ${ }^{29}$ Der Herr ist damit nicht mehr auf die Anerkennung durch den Knecht angewiesen - dieser aber vielleicht immer noch auf seine. Das macht deutlich, dass, sobald die Szene der Anerkennung gesellschaftlich gedacht wird, sie nicht mehr zwangsläufig auf

25 Vgl. Habermas, Jürgen, Theorie des kommunikativen Handelns, 2 Bde., Frankfurt / Main 1995 oder Apel, Karl-Otto, Diskurs und Verantwortung. Das Problem des Übergangs zur postkonventionellen Moral, Frankfurt / Main 1990.

26 Kojève, Alexandre, Hegel. Kommentar zur Phänomenologie des Geistes. Mit einem Anhang: Hegel, Marx und das Christentum, Frankfurt / Main 1975 (franz. Original 1947), S. 64.

27 Ebd.; Tugendhat, Ernst, Selbstbewusstsein und Selbstbestimmung. Sprachanalytische Interpretationen (1979), Frankfurt / Main, 6. Aufl., 1997, S. 341.

28 Vgl. dazu etwa Siep, Ludwig, Anerkennung als Prinzip der praktischen Philosophie. Untersuchungen zu Hegels Jenaer Philosophie des Geistes, Freiburg u. a. 1979, vor allem seinen Exkurs über »Zweier- und Dreierbeziehungen in der Sozialphilosophie des 20. Jahrhunderts«, S. 76-85.

29 Todorov, Tzvetan, Abenteuer des Zusammenlebens. Versuch einer allgemeinen Anthropologie, Frankfurt / Main 1998, S. 36 ff. 
Symmetrie ausgelegt ist, sondern, dass die Begegnung zwischen Ich und Du auf einer grundlegenden Asymmetrie aufbauen kann. Durch das Auftauchen des Dritten kann die wechselseitige Angewiesenheit auf Anerkennung zwischen Herr und Knecht einer einseitigen Abhängigkeit weichen.

Diese Abhängigkeit vom Andern hat Folgen für den Begriff der Anerkennung. In der Alltagssprache und auch im philosophischen oder sozialwissenschaftlichen Diskurs wird >Anerkennungく oft im Sinne von Bestätigung, Wertschätzung, Achtung oder Bewunderung verwendet, womit ein Vorgang oder eine Haltung bezeichnet ist, durch die Menschen ihrem Gegenüber eine positive Bewertung kundtun. Auch Axel Honneth gründet seine Anerkennungstheorie auf diesem Gedanken, wenn er schreibt, dass »Anerkennung in ihrer elementaren Form eine expressive Geste der Befürwortung $\aleph^{30}$ ist. Anerkennung kann jedoch auch Unterwerfung bedeuten. Das zeigt sich, sobald die Szene der Anerkennung als gesellschaftliche Szene, als Szene unter Dritten verstanden wird. Der Knecht steht hier in einer einseitigen Abhängigkeit, er ist auf die Anerkennung, die ihm sein Herr gibt angewiesen. Damit ist er nicht nur für jene Formen der Ansprache offen, die eine Befürwortung zum Ausdruck bringen, sondern er ist auch jenen Formen der Ansprache ausgeliefert, die auf eine Unterwerfung abzielen. Denn selbst noch eine verletzende Ansprache ist eine Ansprache, die die Angesprochene als soziales Wesen anerkennt. Oder anders herum gesagt: Weil jede Ansprache durch eine Andere uns ein soziales Leben zugesteht, sind wir auf deren Anerkennung auch dann angewiesen, wenn sie in einer demütigenden, beleidigenden oder herabwertenden Ansprache besteht. Die Asymmetrie im Verhältnis zur Andern zeigt in grellem Licht, dass auch Formen der Ansprache, die auf Unterwerfung zielen, noch Formen der Anerkennung sind, einfach aufgrund der Tatsache, dass sie ihr Gegenüber überhaupt ansprechen und als soziales Wesen zur Kenntnis nehmen.

Zugespitzt lässt sich in dieser Perspektive umgekehrt auch sagen, dass selbst bestätigende, wertschätzende Anerkennungsakte, wie ein Lächeln, ein Lob oder ein Zuspruch, eine Unterwerfung unter die Andere beinhalten. Denn sie führen mir vor Augen, wie sehr ich von der Anderen abhänge. Nicht nur missachtende Formen der Anerkennung, sondern jegliche Anerkennungsakte sind folglich im Grunde genommen als Unterwerfung zu verstehen. Diese Einsicht ist in den zwei Bedeutungsdimensionen des Englischen >to be subject to somebody< aufgespeichert: >Für jemanden ein soziales Wesen zu sein` ist hier gleichbedeutend mit >jemandem unterworfen zu sein<. In der verletzenden Ansprache tritt also lediglich mit aller Deutlichkeit eine Abhängigkeit vom Anderen zu Tage, die jeder Form der Ansprache unterliegt. Diese grundlegende Asymmetrie in der Struktur der Intersubjektivität bedeutet jedoch nicht, die unendliche Aufgabe eines Durcharbeitens oder Umarbeitens dieser Asymmetrie zu verabschieden. Im Gegenteil,

30 Honneth, Axel, »Unsichtbarkeit. Über die moralische Epistemologie von >Anerkennung «, in: ders., Unsichtbarkeit. Stationen einer Theorie der Intersubjektivität, Frankfurt / Main 2003, S. 10-27, hier S. 21. 
durch den Aufweis dieser Asymmetrie zeigt sich erst, in welchem Ausmaß eine Umarbeitung dieser Asymmetrie eine schwierige, aber umso dringlichere Aufgabe ist.

\section{Symbolische Verletzbarkeit und sozialer Tod}

Worte können beschmutzen. Sie können die Ehre `beflecken`, wie wir sagen. Beleidigt worden zu sein, bedeutet daher oftmals, sich gezwungen zu sehen, den eigenen Namen >reinwaschen $>$ zu müssen. ${ }^{31}$ Diese Reinwaschung erfordert unter bestimmten Umständen, die Unversehrtheit des sozialen Seins über die Unversehrtheit des physischen Seins zu stellen. Sie kann dazu führen, dass die Angesprochene lieber den eigenen Tod riskiert als eine bestimmte Demütigung zu ertragen. Das ist beispielsweise im Duell der Fall: Eher den eigenen Tod riskieren als mit einer befleckten Ehre weiter leben, so könnte die Maxime desjenigen lauten, der den Kampf auf Leben und Tod eingeht. ${ }^{32}$ Herabwertende Äußerungen sind in diesem Szenario alles andere als >bloße Worte`, sie verweisen vielmehr auf eine dramatische Verletzbarkeit durch Worte, eine Verletzbarkeit, die scheinbar so groß ist, dass für sie die physische Existenz aufs Spiel gesetzt wird. Worin nun liegt diese Verletzbarkeit? Was macht ihre Tragik aus? Welche Logik liegt ihr zugrunde?

Durch die Sprache verletzt zu werden bedeutet, auf einen randständigen sozialen Ort verwiesen zu werden. >Herabsetzung〈, >Erniedrigung〈oder >Abwertungく etwa bringen zum Ausdruck, dass jemand an einem minderwertigen, prekären Ort im Sozialen positioniert worden ist - zumindest temporär oder in bestimmten Kontexten. Folgen wir diesem Alltagsvokabular, dann können wir davon sprechen, dass sprachliche Gewalt gemäß einer Logik sozialer Ortsverschiebungen arbeitet. Diese Logik hat eine zentrifugale Ausrichtung, sie zieht ihre Adressatin an den sozialen Rand hin. Die Verletzung durch die Ansprache weist der Angesprochenen zwar einen Platz zu: aber es ist ein minderwertiger, zunehmend unsicherer Platz.

Wie prekär eine randständige Platzierung ist, zeigt sich beispielsweise in der Tatsache des Angesprochenwerdens. Denn gilt der eigene Ruf als beschmutzt, dann drohen sich die Anderen an mir >die Finger schmutzig zu machen kann ein zunehmender Verlust der Ansprache folgen, der sich darin zeigt, dass ich gemieden werde. Der Situation der Ansprache wird ausgewichen, indem einer Konfrontation in der face-to-face Situation ausgewichen wird. Ein Umstand, der in der Aussage $>$ Wer will schon mit so einer wie dir etwas zu tun ha-

31 Vgl. dazu etwa Benedict, Ruth, Chrysantheme und Schwert, Frankfurt / Main 2006, (engl. Original 1946), vor allem Kapitel 8: `Seinen eigenen Namen reinwaschen`, S. 131-158.

32 Das hat Hegel in seinen Überlegungen zum Kampf auf Leben und Tod eindrücklich deutlich gemacht: Hegel, Georg Wilhelm Friedrich, Phänomenologie des Geistes (1807), in: ders., Werke 3, Frankfurt / Main 1986, S. 148 ff. 
ben ganz explizit wird. Die Ansprache kann jedoch auch in meiner Gegenwart ausbleiben, und sich darin zeigen, dass ich übergangen werde. Etwa in der Art, dass die Umstehenden nach ihrer Meinung oder ihrem Rat gefragt werden, ich jedoch nicht. Diese Bedeutungslosigkeit kommt in der Aussage $>$ Von dir will niemand etwas wissen zum Ausdruck. Das eigene Sein gilt so wenig, dass es gar nicht erst adressiert wird. Auf der anderen Seite kann soziale Randständigkeit auch in dem Umstand zum Ausdruck kommen, dass die Betroffenen immer weniger die Möglichkeit haben, selbst diejenigen zu sein, die andere ansprechen und eine Antwort hervorrufen können. Der Verlust dieser sprachlichen Handlungsfähigkeit kann sich darin zeigen, dass mit zunehmender Desintegration ganz grundlegend die Möglichkeiten zur Rede immer stärker genommen werden: ${ }^{33}$ Es gibt erst gar keine Möglichkeit, dass die Stimme Gehör findet. Das mag daran liegen, dass eine Person nicht gefragt wird, dass ihr gedroht wird, bloß >das Maul zu halten ২ oder dass sie einfach >nichts zu melden` hat. Oder man erhält keinen Zugang zu den Orten, an denen eine Stimme überhaupt gehört werden kann. Der Stimmverlust kann aber auch darin bestehen, dass sprachliche Handlungen einfach keine Effekte mehr erzielen, da ihnen die nötige Autorität zur Verwirklichung ihrer Rolle fehlt. Verliert man eine überlegene soziale Position und gerät in eine zunehmende soziale Unterlegenheit, ist es zwar noch möglich, eine Äußerung zu vollziehen - aber sie kann ins Leere laufen, keine Kraft mehr haben oder nichts mehr zählen. Es mag der sprechenden Person mit Worten einfach nicht mehr gelingen, bestimmte Effekte hervorzurufen, weil sie nicht die nötige Autorität zum Sprechen besitzt. Unser abfälliges \Lass sie doch reden, interessiert ja eh keinen bringt diese Handlungsohnmacht zum Ausdruck.

Mit der Sprache kann man also der Sprache berauben: die Sprache kann ins Schweigen sprechen. Dieses Schweigen kann soweit gehen, das wir ganz aus dem Spiel von Ansprache und Antwort ausgeschlossen, nicht mehr als soziales Wesen adressiert werden und damit die soziale Existenz vollkommen verlieren. Wir sprechen in diesem Fall vom ssozialen Tod . Die Positionierung am sozialen Rand ist oft nur einen Fußbreit von diesem entfernt oder tendiert dazu, schon im nächsten Moment in diesen überzugehen. Zwischen sozialer Teilhabe und sozialem Tod gibt es keine klaren Trennlinien, sondern eher eine bedrohliche Übergängigkeit. Die verletzende Sprache kann auf einen sozialen Ort sprechen, der sich als ein Nicht-Ort entpuppt. ${ }^{34}$ Ein Ort, der keine Gewissheit darüber zulässt, ob wir noch Teil des Sozialen sind.

Sozial ausgeschlossene Personen werden behandelt, als seien sie $>$ Luft $<$, wie beispielsweise Obdachlose. Meist werden sie von Passanten einfach gar nicht bemerkt, an ihnen wird vorübergegangen wie man an einem Baum vorübergeht oder man irgendeinem Gegenstand ausweicht. Dem Obdachlosen wird auf seine

33 Wir orientieren uns hier an Rae Langtons Typologie der >unsprechbaren Akte،. Vgl. dies., »Sprechakte und unsprechbare Akte«, in diesem Band, S. 107-146.

34 Butler, Hass spricht, a.a.O., S. 12. 
Frage nach etwas Kleingeld oft nicht nur keine materielle Gabe entgegengebracht, sondern manchmal noch nicht einmal geantwortet. In diesem Sinn erfahren Obdachlose überhaupt keine oder kaum noch eine Adressierung von Anderen, und darüber hinaus haben Obdachlose freilich auch ganz buchstäblich keine Adresse mehr: Sie sind ohne bauliches wie auch ohne soziales Obdach. ${ }^{35} \mathrm{Im}$ sozialen Tod ist der Stimmverlust so drastisch, dass die Fähigkeit der Betroffenen, mit Anderen zu kommunizieren, ganz verloren geht. Diese Art des Stimmverlusts führt zum Abbruch der Kommunikation, oder, wie Petra Gehring es ausdrückt, mündet in einen $>$ Kommunikations-Tod $<{ }^{36}$ Auch wenn dieser Tod keinen Leichnam hinterlässt, bringt er doch etwas hervor, was wir vom physischen Tod her kennen: Totenstille. So wie die körperlich Tote nicht mehr spricht, kann auch die sozial Tote nicht mehr sprechen - sie ist verstummt. Die lebende Tote vermag sprachlich kein soziales Band mehr mit anderen Menschen zu knüpfen und bleibt vom sozialen Leben ausgeschlossen. Ein letztes Aufbegehren gegen den Kommunikations-Tod stellt oft die Flucht in die Provokation dar. Provokation meint dann ganz wörtlich pro-vocare, den Ruf nach der Stimme des Anderen, nach seiner Antwort. Wer sich als unsichtbar erlebt, als jemand, der wie Luft behandelt wird, der versucht oft, eine Antwort zu provozieren - mit jeglichen Mitteln, und indifferent gegenüber der Art der Antwort. ${ }^{37}$ Personen beginnen dann, andere zu beleidigen, sie zu bedrohen, sie anzurempeln, nur um die Gewissheit zu erhalten, jemand zu sein, dessen Tun auf irgendeine Resonanz bei der Anderen stößt.

\section{Die Grammatik sprachlicher Gewalt}

Von der Frage, warum Worte verletzen können, wollen wir nun zur Frage übergehen, wie Gewalt durch Sprache ausgeübt wird. Mit Hilfe der >Grammatik sprachlicher Gewalt $<$ werden wir die verletzende Rede dabei als eine soziale Praxis rekonstruieren. Das heißt Folgendes: (i) Keine Äußerung als solche verletzt zwangsläufig - und zugleich kann beinahe jede beliebige Äußerung verletzen. Selbst das schwerste Schimpfwort muss nicht notwendig zur Herabsetzung des Angesprochenen führen (ein Schwarzer, der einen Weißen als >Scheiß-Weißen< beschimpft, wird vor erheblichen Schwierigkeiten stehen). Und selbst noch ein Lob vermag abzuwerten: etwa in der Formulierung >Für eine Frau können Sie wirklich gut Autofahren . Das bedeutet, dass keine rein linguistische Untersu-

35 Vgl. dazu Bourdieu, Pierre, Meditationen. Zur Kritik der scholastischen Vernunft, Frankfurt / Main 2001, S. 172-199.

36 Vgl. Gehring, Petra, »Die Wiederholungs-Stimme. Über die Strafe der Echo«, in: Doris Kolesch / Sybille Krämer (Hg.), Stimme, Frankfurt / Main 2006, S. 85-110.

37 Vgl. dazu Axel Honneths Anknüpfung an Ralph Ellisons Roman Der unsichtbare Mann, in dem der schwarze Protagonist Passanten auf der Straße ostentativ anrempelt oder um sich schlägt, um dem Gefühl zu entgehen, unsichtbar zu sein (Honneth, »Unsichtbarkeit«, a.a.O., S. 14 f.). 
chung allein etwas über das Gewicht einer Missachtung aussagen kann. Entscheidend sind vielmehr die Umstände der Äußerung - wer zu wem was in welchem Kontext sagt. Die Grammatik sprachlicher Gewalt ist daher keine Grammatik des Sprechakts, sondern eine Grammatik des Äußerungsszenarios. (ii) Die Szene der Äußerung bezieht sich nicht primär auf singuläre, rein individuelle Umstände: zum Beispiel darauf, dass die Sprecherin und die Adressatin schon am Tag zuvor miteinander Streit hatten, und die Adressatin deshalb das Lob der Sprecherin >in den falschen Hals $<$ bekommt. Sie bezieht sich vielmehr auf soziale Machtverhältnisse. Die Grammatik sprachlicher Gewalt sucht die Kraft einer Äußerung daher in den Kräfteverhältnissen des Sozialen. (iii) Die Verletzungsmacht der Rede wird oftmals ganz von der subjektiven Empfindlichkeit der Angesprochenen her verstanden. Die Angesprochene, so behauptet diese Position, reagiere aufgrund ihres biographischen Hintergrunds auf ein bestimmtes Wort emotional. Die Ansprache ist in dieser Sicht Projektionsfläche für innerpsychische Konflikte der Betroffenen, und die Verletzung wird zu einer Deutungsleistung der Angesprochenen. ${ }^{38}$ Die Äußerung in ihrer sozialen Logik zu untersuchen, bedeutet jedoch, sprachliche Gewalt aus dem Reich des Subjektiven zu holen. Für die Verletzungskraft sprachlicher Gewalt ist nicht konstitutiv, was auf einer psychischen Ebene geschieht, sondern was die Äußerung mit der Angesprochenen im Sozialen tut. (iv) Sprachliche Gewalt als soziale Praxis zu verstehen, bedeutet schließlich auch, den verletzenden Sprechakt als kommunikativen Akt zu denken, d. h. als Akt, der sich an jemanden richtet, der auf die Ansprache antworten kann. Die Grammatik sprachlicher Gewalt untersucht daher zugleich auch die Bedingungen, unter denen eine Antwort gegeben und die Gewalt eventuell aufgefangen oder erwidert werden kann.

\section{Sprachliche Gewalt als Absetzungsakt}

Gewalt ist zerstörerisch - und gewiss ist auch sprachliche Gewalt zerstörerisch; sie setzt herab und entzieht ihrer Adressatin ihren sozialen Platz. Doch sie verstößt die Angesprochene von ihrem Platz, um ihr einen anderen, untergeordneten Platz zuzuweisen. Dieses Wissen ist in vielen Begriffen, die wir für die Beschreibung sprachlicher Gewalt benutzen aufgehoben: Herabsetzung, Erniedrigung oder $A b$ wertung etwa bringen zum Ausdruck, das jemand seine sozialen Stellung in der Welt verloren hat. Sie nennen den Entzug einer bisherigen sozialen Stellung, einen Fall - doch dieser Fall ist kein freier Fall, sondern er endet an einem anderen Platz, den wir als unterlegenen, untergeordneten, unterworfenen Platz beschreiben. Nehmen wir das topologische Vokabular ernst, das wir in unseren Beschreibungen sprachlicher Gewalt anwenden, dann können diese Worte als Hinweis darauf verstanden werden, dass sprachliche Gewalt sowohl als eine Kraft zur Zerstörung einer bestehenden Ordnung betrachtet werden kann, als

38 Vgl. Nunner-Winkler, Gertrud, »Mobbing und Gewalt in der Schule. Sprechakttheoretische Überlegungen«, in: Westend, Heft 1, 2004, S. 91-100, hier S. 94. 
auch als eine Kraft, die neue Ordnung schafft. Anders ausgedrückt: Sprachliche Gewalt muss nicht nur eine destruktive, sondern kann auch eine produktive Kraft sein - sie kann `negative〈 Ordnung stiften, und zwar in Form der >Unter-Ordnung einer Person. Der tieferliegende Grund dafür besteht darin, dass jede Ansprache die Angesprochene unvermeidlich ins Soziale einführt. Das lässt sich auch so formulieren: Mit einer Sprache, die ihre Adressatin immer an einem sozialen Ort anspricht, ist es nicht möglich, dieser ihren sozialen Ort ganz zu rauben - es kann allein ein anderer, minderwertiger Ort konstituiert werden. Sprachliche Gewalt beruht daher nicht in erster Linie auf einer Logik der Zerstörung, als vielmehr auf einer sozialen Logik der Ortverschiebung.

Als Paradigma sprachlicher Gewalt können Absetzungsakte gelten. In ihnen geht es darum, wie Bourdieu sagt, jemandem mitzuteilen, »er habe diese oder jene Eigenschaft, und zugleich, er habe sich der ihm auf diese Weise zugesprochenen sozialen Natur gemäß zu verhalten « ${ }^{39}$. Ein Beispiel für einen Absetzungsakt ist etwa das militärische Degradierungszeremoniell, in dem ein Offizier zum einfachen Gefreiten zurückgestuft wird. Der Absetzungsakt verändert die Stellung der adressierten Person im Sozialen. Er verändert die Vorstellung, die die abgesetzte Person von sich selber hat, und auch das Verhalten, zu dem sich die Person nun verpflichtet fühlt. Und er verändert die Vorstellung, die Andere von nun an von der abgesetzten Person haben, und das Verhalten, das sie der Person gegenüber nun an den Tag legen. Die Absetzung ist ein Akt >sozialer Magie «: Ohne dass jemandem physisch etwas zugefügt würde, verwandelt sich der soziale Status einer Person - dazu nötig sind: nur Worte.

Im Horizont von Absetzungsakten zeigt sich, dass die Gewaltsamkeit sprachlicher Gewalt auf einem Doppelcharakter beruht, der im deutschen Begriff >Gewalt « aufgehoben ist. \Gewalt « meint auf der einen Seite die verletzende Gewalt, die wortgeschichtlich auf das lateinische vviolentia zurückgeht. ${ }^{40}$ Bei ihr handelt es sich um Gewalt als Kraft; sie wird gemessen an ihrer Wucht oder Stärke. Gewalt ist in dieser Hinsicht gleichbedeutend mit Schädigung, Verwüstung oder Zerstörung. Auf der anderen Seite bezieht sich Gewalt jedoch auch auf eine verfügende Gewalt, die auf das lateinische >potestas zurückgeht und in Ausdrücken wie etwa >Staatsgewalt $\iota,>$ Amtsgewalt $<$ oder >geistliche Gewalt auftritt. Gewalt wird in dieser Dimension gemessen an dem Vermögen oder dem Können, etwas geschehen zu lassen; kurz: es handelt sich um Gewalt als Macht. Geht man nun davon aus, dass sprachliche Gewalt nicht nur einfach destruktiv, sondern auch produktiv ist, indem sie ihr Gegenüber als ein unterlegenes anspricht, dann wird deutlich, dass sich sprachliche Gewalt nicht im Aspekt der violentia erschöpft, sondern diese ein Produkt der potestas ist. Verletzend ist Sprache in dem Maße, wie es ihr gelingt, ihr Gegenüber mit Macht als untergeordnet, als herabgesetzt

39 Bourdieu, Was heißt sprechen? a.a.O., S. 99.

40 Vgl. etwa Imbusch, Peter, »Der Gewaltbegriff«, in: Wilhelm Heitmeyer / John Hagan (Hg.), Internationales Handbuch der Gewaltforschung, Wiesbaden 2002, S. 2657, hier S. $29 \mathrm{ff}$. 
oder als erniedrigt einzusetzen. Zugespitzt formuliert heißt das: Die verletzende Gewalt beruht im Grunde genommen auf einer verfügenden Gewalt. Erst indem jemand die verfügende Gewalt (Macht) ausübt, eine Person herabzusetzen, wendet er ihr gegenüber eine verletzende Gewalt (Kraft) an.

Um einen Menschen physisch zu verletzen oder zu töten, bedarf es keiner verfügenden Gewalt, keiner Macht. Denn wenn wir von der Macht zu töten reden, dann ist von einer Macht die Rede, die allen Menschen zukommt (einen Messerstich kann tendenziell jede jeder zufügen) - sie stellt keine spezifische Verfügungsgewalt mehr dar. ${ }^{41}$ Physische Gewalt kann zwar durch Macht legitimiert werden, aber ihre Ausübung erfordert letztlich nur Kraft - sei es in Form der eigenen Körperkraft oder in Form einer durch den Einsatz von Waffen angeeigneten Durchschlagskraft. Im Gegensatz zu physischer Gewalt ist sprachliche Gewalt jedoch auf Macht angewiesen. Das verdeutlichen Absetzungsakte, denn die Verwandlung, auf die sie abzielen, hat nur Erfolg, wenn sie mit Macht auferlegt wird. Eine solche Macht - das hat Austin mit der Entdeckung seiner >ursprünglichen Performativa aufgezeigt - liegt weder in der Semantik noch in der Physis der Äußerung. Sie kommt vielmehr gleichsam von außen, vom Sozialen her: Performativa verweisen immer auf eine gesellschaftliche Szene der Äußerung. ${ }^{42}$ Den Bedingungen, unter denen sprachliche Absetzungsakte sich mit Macht zu sättigen vermögen, wollen wir im nächsten Schritt genauer nachgehen.

\section{Figuren des Dritten}

Absetzungsakte sind rituelle Akte, die ihre Kraft aus gesellschaftlichen Kräfteverhältnissen beziehen. Weniger die Intention einer Sprecherin als die sozialen Bedingungen einer Äußerung müssen daher bei der Untersuchung sprachlicher Gewalt im Vordergrund stehen. Garfinkel schlägt in diesem Sinne vor, das Gerichtszeremoniell zwischen der Anklägerin, der Angeklagten und den Geschworenen als `Urszene< von Absetzungsakten zu begreifen. ${ }^{43}$ Wir greifen diese trialogische Anordnung auf und gehen davon aus, dass Absetzungsakte nicht auf einem dualen Verhältnis von Ich und Du beruhen, sondern durch eine dritte Instanz vermittelt sind. Während die Sprachphilosophie Kommunikation gemeinhin im Horizont eines dialogischen Paradigmas betrachtet, wird sich am Beispiel von sprachlicher Gewalt auf plastische Weise zeigen, wie sehr sich Kommunika-

41 Popitz formuliert diesen Umstand so: »Weil Menschen andere Menschen töten können, ist alle Macht von Menschen über Menschen unvollkommen." (Popitz, Heinrich, Phänomene der Macht, 2., stark erw. Aufl., Tübingen 1992, S. 57)

42 Austin, John L., Zur Theorie der Sprechakte (How to Do Things With Words), 2. Aufl., Stuttgart, 1979 (engl. Original 1962).

43 Garfinkel, Harold, »Bedingungen für den Erfolg von Degradierungszeremonien«, in diesem Band, S. 49-57 (engl. zuerst 1956). 
tion gerade mittels eines Dritten vollzieht. Sprachliche Gewalt muss folglich in grundlegender Weise eine »Gewalt unter Dritten $\aleph^{44}$ verstanden werden.

(i) Der Zeuge als personaler Dritter: Eine `dritte Instanz^ finden wir zunächst ganz plastisch in dritten Personen, an die sich viele Beleidigungen wenden und die den performativen Akt bezeugen sollen. Das machen semi-direkte Sprechakte ${ }^{45}$ besonders deutlich: Sie wenden sich nicht an jemanden, sondern sprechen zu einem Publikum über jemanden. Ist diejenige, über die gesprochen wird, sogar noch anwesend, wird der im Gesagten ausgedrückte Ausschluss auch noch sprachlich performiert. Solche semi-direkten Sprechakte, die sich ganz von ihrer Adressatin abwenden, machen deutlich, dass die Dritte die eigentliche Adressatin des verletzenden Sprechens ist. Die Zuschauer sind dabei nicht in erster Linie als Individuen anwesend, sondern als buchstäbliche Verkörperungen des Sozialen. Im Gegensatz zu sprachlicher Gewalt hinterlässt physische Gewalt, wie etwa der Schlag mit der Faust, schmerzhafte Spuren am Körper des Opfers. Wo keine materiellen Spuren von der Gewalt Zeugnis geben, ist die Anwesenheit einer Zeugenschaft umso gewichtiger. Was in dialogischer Kommunikation als Anspielung übergangen werden kann, droht in einer öffentlichen Situation plötzlich eine eigentümliche Kraft zu entfalten, die von der Hörerin kaum ignoriert werden kann. Darum reden wir auch davon, dass jemand >vor allen Leuten ‘ bloßgestellt wurde. Und darum ist es so entscheidend, ob wir mit unserem verächtlichen Witz die >Lacher auf unserer Seite haben< oder nicht. ${ }^{46}$

Garfinkel hat darauf aufmerksam gemacht, dass es nicht immer genügt, dass das Publikum eine Situation be-zeugt, es muss oft auch von einer Äußerung überzeugt werden. ${ }^{47}$ Garfinkel rückt mit dieser Überlegung die zeitliche Verstetigung der Absetzung in den Mittelpunkt. Wäre das Publikum immer auf die Rolle des Zeugen reduzierbar, dann würde seine Rolle stets nur darin bestehen, möglichst neutral zu bestätigen, dass eine Sprechhandlung $\mathrm{x}$ von einer Person A an eine Person B adressiert worden ist. Damit wäre diese Handlung aber noch nicht verstetigt. Denn damit eine Missachtung ihre Kraft entfaltet, kommt es für die Sprecherin nicht nur darauf an, dass ihr Sprechen als ein missachtendes Sprechen

44 Liebsch, Burkhard, »»Sprechende〈 Gewalt«, in: Kristin Platt (Hg.), Reden von Gewalt, München 2002, S. 150-174, hier S. 163.

45 Der Terminus >semi-direkte Sprechakte mann, Carl F., »Verbal Discrimination: A Neglected Chapter in the Social Psychology of Aggression«, in: Journal for the Theory of Social Behaviour, Heft 1, Bd. 28, 1998, S. 41-61, hier S. 54.

46 Diejenige, die über jemanden spottet, versucht, so Freud, »die ursprünglich störende dritte Person zum Bundesgenossen« zu machen (Freud, Sigmund, Der Witz und seine Beziehung zum Unbewussten (1905), in: ders., Der Witz und seine Beziehung zum Unbewussten. Der Humor, 6. Aufl., Frankfurt / Main 2001, S. 114.). Dieser Umstand spiegelt sich auch darin wieder, dass laut Strafgesetzbuch für die Schwere einer ehrkränkenden Beleidigung der Grad an Öffentlichkeit mit ausschlaggebend ist; vgl. Sornig, Karl, »Beschimpfungen«, in: Grazer Linguistische Studien. Sprache \& Gesellschaft, Heft 1, 1975, S. 150-170, S. $164 \mathrm{f}$.

47 Garfinkel, »Bedingungen für den Erfolg«, a.a.O., S. 53 ff. 
wahrgenommen wird, sondern dass die adressierte Person in den Augen des Publikums tatsächlich zu einer missachtenswerten Person wird. Das wird sie aber oft erst dadurch, dass das Publikum eine Sprechhandlung nicht nur bezeugt, sondern von ihr überzeugt wird. Und um überzeugt zu werden, muss das Publikum die Äußerung der Worte nicht nur beglaubigen, sondern es muss ihr zugleich auch seinen Glauben schenken. Erst dadurch erhält die Demütigung eine Dauerhaftigkeit, durch welche sie sich über die Zeit hinweg wirkungsvoll in das Soziale einschreiben kann.

(ii) Autoritative Sprecherpositionen: Eine autoritative Sprecherposition einzunehmen bedeutet, nicht in erster Linie als Individuum zu sprechen, sondern mit der Stimme von Dritten. Die autorisierte Sprecherin kann mit Worten auf Andere einwirken, weil sich in ihrer Äußerung die symbolische Kraft einer ganzen Gruppe konzentriert. Die »delegierte Macht« verleiht der eigenen Stimme Gewicht. $^{48}$ Diejenige, die z. B. im Namen des Gesetzes, im Namen der Wissenschaft oder im Namen einer Behörde spricht, macht sich die ganze Kraft einer Institution zu eigen. »Die Wirkung des performativen Diskurses, der den Anspruch erhebt, das Gesagte mit dem Akt des Sagens herbeizuführen, ist so groß wie die Autorität dessen, der spricht. ${ }^{49}$ Die autorisierte Sprecherin spricht folglich in gewissem Maße immer mit einer fremden Stimme, der Stimme der institutionalisierten Macht. Diejenige, die in ihrer sozialen Stellung genügend Autorität akkumuliert hat, kann sich des Erfolgs ihrer Missachtung weitgehend sicher sein; diejenige dagegen, die nicht über einen solchen Rückhalt verfügt, muss viel eher damit rechnen, mit ihrer Missachtung zu scheitern. Der Manager, der aus dem Supermarkt kommend den Obdachlosen verächtlich als >Schnorrer bezeichnet, wird seiner Äußerung mehr Kraft verleihen können als der Obdachlose, der umgekehrt den Manager einen >Schnöselく nennt. Ein noch drastischeres Beispiel sind Sklaven, die in vielen Gesellschaften nicht dazu in der Lage waren, ihre Herren mit Worten zu verletzen - ihre Äußerungen blieben einfach bedeutungslose Geräusche. ${ }^{50}$

(iii) Gesellschaftliche Klassifikationen: Sprachliche Gewalt gewinnt ihre Kraft durch das Aufrufen gesellschaftlicher Klassifikationen, die sich in einem hegemonialen Diskurs sedimentiert haben - sie muss sich also mit dritten Stimmen überlagern. ${ }^{51} \mathrm{Zu}$ den Klassifikationen, die sprachliche Gewalt wieder aufrufen kann, gehören binär strukturierte Kategorien, in denen der jeweils sekundäre Terminus den abgewerteten, unterlegenen Platz markiert: weiß / schwarz, maskulin /

48 Zur >delegierten Macht< und zum autoritativen Sprechen, vgl. Bourdieu, Was heißt sprechen?, a.a.O., S. 99-109; Austin, Zur Theorie der Sprechakte, a.a.O., S. 35-55.

49 Bourdieu, Was heißt sprechen?, a.a.O., S. 125.

50 Vgl. Patterson, Orlando, Slavery and Social Death. A Comparative Study, Cambridge u. a. 1982, S. 82.

51 Darauf hat besonders Judith Butler im Kontext der hate speech-Debatte mit ihren Überlegungen zum Rezitieren rassistischer Äußerungen aufmerksam gemacht (vgl. etwa Butler, Hass spricht, a.a.O., S. 53 ff.). 
feminin, eigenes / fremdes, normal / behindert usw. Solche Ungleichheitssemantiken sind durch die Geschichte einer Gesellschaft geprägt worden. Die Kraft und Legitimität der performativen Äußerung wird nicht im Augenblick geboren, sondern sie resultiert aus einer sedimentierten Geschichtlichkeit, aus dem, was durch Traditionen, Bräuche und Etiketten überliefert ist. Um dem Sprechen verletzende Kraft zu verleihen, können solche Klassifikationen aufgerufen und angeeignet werden. Die verletzende Äußerung wird daher in einem gewissen Sinne nie nur gesagt, sondern immer wieder-gesagt. Nicht die Sprecherinnen verfügen über die Macht der verletzenden Benennung, sondern ein historischer Chor an Stimmen: Gesellschaftliche Klassifikationen tragen die kollektive Kraft einer ganzen Kultur in sich.

Die Autorität der sprechenden Person verleiht der Äußerung Gewicht, die Klassifikation gibt der verletzenden Formel Kraft und die Anwesenheit des Publikums verbürgt den dauerhaften Vollzug des sprachlichen Gewaltaktes. Für Missachtungen gilt folglich, dass sich soziale Kräfte in einer Stimme verdichten müssen, damit der performative Akt seine Gewalt - im doppelten Sinne - entfalten kann. Die Stimme der Einzelnen gewinnt in der Perspektive der Grammatik sprachlicher Gewalt nur in der Übereinstimmung mit dem kumulativen Gewicht einer Vielzahl von anderen Stimmen ihre Verletzungsmacht.

Dennoch kann keine der drei Figuren des Dritten das Gelingen des Gewaltaktes abschließend sichern. Die unterschiedlichen Weisen des Bezugs auf einen Dritten gewährleisten den Erfolg der gewaltsamen Äußerung nie vollkommen. Und die drei Figuren des Dritten decken das Feld der Möglichkeiten, sprachliche Gewalt auszuüben, in keiner Weise vollständig ab. Der Erfolg einer Missachtung ist beispielsweise nicht zwangsläufig an ein Publikum gebunden. Vielmehr gilt oft das genaue Gegenteil: Für viele verletzende Äußerungen scheint nicht die Öffentlichkeit, sondern die Privatsphäre den Ausgangspunkt zu bilden. In engen persönlichen Bindungen können Worte so sehr treffen wie in kaum einem anderen sozialen Kontext. Verletzende Äußerungen zielen in dieser Konstellation nicht unbedingt darauf ab, das Gesagte öffentlich bekannt zu machen, sie ziehen ihre Wirkmächtigkeit vielmehr aus der intimen Kenntnis der Anderen. Oft kommen anwesende Dritte deshalb mit der Subtilität der Äußerungen gar nicht mehr mit; es mangelt ihnen am notwendigen Hintergrundwissen, durch das eine mehrdeutige Anspielung der Sprecherin auf einen `wunden Punkt $\measuredangle$ ihrer Partnerin oft erst ihren Sinn erhält. Eine solche Konstellation der Verletzungsmacht in engen Bindungen beruht freilich auch auf einer starken Abhängigkeit der adressierten Person von der Sprecherin, eine Situation, in dem sie sich dieser nicht ohne Weiteres entziehen kann. Diese grundlegende Form der Asymmetrie, die auf mehr oder weniger drastische Weise alle Beziehungen zwischen Selbst und Anderer prägt, tritt in intimen Bindungen besonders deutlich zutage. In intimen Beziehungen begeben sich das Selbst und die Andere in eine leidenschaftliche Verletzlichkeit, die auf latente Weise für Intersubjektivität als solche kennzeichnend ist. Es ist diese Abhängigkeit von der Anderen, die uns in engen Zweierbeziehungen 
- vollkommen unabhängig von der Frage, ob Dritte anwesend sind oder auch nicht - extrem verletzbar macht.

Auch hebeln sich die unterschiedlichen Gestalten des Dritten oft gegenseitig aus. Eine Person mit großer Autorität etwa, deren Machtposition gesellschaftliche Anerkennung genießt, kann von Äußerungen verwundet werden, die mit der Instanz des Dritten in Gestalt von Diskursen und Klassifikationen spricht. Wenn beispielsweise eine Schülerin ihre Lehrerin beleidigt, so muss sie dabei nicht zwangsläufig den Kürzeren ziehen, vielmehr kann es ihr auch gelingen, ihre Missachtung sehr wirkungsvoll zu platzieren, indem sie es so einrichtet, dass sie vor der Schulklasse als diejenige auftritt, die die Lehrerin im Namen der von der Klasse geteilten Werte - etwa: >Unterwerfe dich nie einer Autorität $<$ - herabsetzt. Wenn eine institutionell schwächer gestellte Person an bestimmte Diskurse anknüpfen kann, dann kann der >delegierten Macht` wirkungsvoll entgegengetreten werden.

Und noch mehr als das: Selbst wenn keinerlei Bezug auf einen Dritten vorhanden ist, kann sprachliche Gewalt vollzogen werden. Die drei Figuren des Dritten beziehen nicht alle möglichen Weisen mit ein, mit denen sprachliche Gewalt ausgeübt werden kann. Es gibt eine unendliche Vielzahl an rhetorisch-kreativen Kniffen, Schachzügen, Spielereien, Tricks, Zwischentönen, die einem jeden Sprechen Verletzungskraft verleihen kann. Kreative Rhetoriken verletzenden Sprechens kommen oft ohne jeden Bezug auf eine der drei Figuren des Dritten aus, sie können allein durch einen situativ-kreativen Umgang mit Worten hervorgebracht werden. Anspielungen, Ironien oder Beiläufigkeiten etwa sind nicht auf bestimmte Inhalte, bestimmte Sprecher oder bestimmte Mitanwesende festgelegt, vielmehr müssen sie in jeder Situation aufs Neue erfunden werden. Sie haben keinen eigenen Ort, von dem aus sie operieren können, vielmehr machen sie sich das vorgegebene Terrain durch taktische Finessen nutzbar.

Allerdings bilden die Figuren des Dritten und die >leisen< Rhetoriken der Verletzung keinen Gegensatz. Im Gegenteil, je stärker eine Sprecherin sich im Verhältnis zur Hörerin mit einer dritten Instanz verschwistern kann, desto subtiler vermag das rhetorische Gewand der Äußerung zu sein. Denn der Umstand, dass die Rolle von sstillen` Beleidigungen konstitutiv unklar ist, bringt für die Sprecherin auch Schwierigkeiten mit sich. Eine vermeintlich belanglose Feststellung, die auf die Abwertung der Adressatin zielt, steht in der Gefahr, tatsächlich als belanglose Feststellung verstanden zu werden. Vielleicht ist die Äußerung für das Publikum überhaupt nicht - und vielleicht auch nicht für die Adressatin - als Missachtung erkennbar. Wenn jedoch eine Sprecherin mit einer Stimme des Dritten sprechen kann, sind die Ohren der Adressatin und des Publikums gemeinhin viel hellhöriger - und das Gelingen der >leisen` Missachtung damit viel sicherer. Der Rückhalt durch Instanzen des Dritten erleichtert folglich den Vollzug sprachlicher Gewalt. Dieser Umstand lässt sich auch negativ wenden: Dritte Instanzen verringern die Gefahr des Scheiterns des Sprechakts. Das ist von besonderer Bedeutung, weil sich das Misslingen sprachlicher Gewaltakte drastisch vom Miss- 
lingen anderer performativer Akte unterscheidet. Sprachliche Gewalt steht - anders als andere Performativa - nicht nur in der Gefahr, snicht zu zünden ${ }^{52}$, sondern sich im Falle ihres Scheiterns gegen ihre Sprecherin zurückzuwenden. Ist sie zu plump vorgebracht, droht die Missachtung als ১fies`, >unangemessen` oder >taktlos` auf ihre Sprecherin zurückzufallen. ${ }^{53}$ Die ungeschriebenen Gesetze der Höflichkeit besitzen in sozialer Hinsicht jedoch ungleiche Geltung: Diejenigen, die wenig gesellschaftliche Autorität besitzen, werden viel leichter in die Grenzen der Höflichkeit verwiesen als diejenigen, die mit der Stimme eines Dritten sprechen.

\section{Die soziale Logik sprachlicher Gewalt}

Sprachliche Gewalt arbeitet gemäß einer Logik der sozialen Ortsverschiebung. Anhand einiger Gegensatzpaare wollen wir im Folgenden die zentralen Mechanismen herausstellen, die bei solchen Verschiebungen zum Einsatz kommen.

Leere und volle Missachtung: >Leere Beleidigungen $<$ bringen die Wut oder den Ärger der Sprecherin zum Ausdruck, sie zielen darauf ab, ihre Adressatin zurückzuweisen und abzuwerten. Ein typisches Beispiel dafür wären zwei Autofahrer, die sich im Straßenverkehr in die Quere kommen und einander als \Idiot< und >Arschloch beschimpfen, um ihrem Ärger >Luft zu machen . Bei solchen Beschimpfungen geht es nicht in erster Linie darum, etwas über das Gegenüber auszusagen, als vielmehr darum, eine abwertende Haltung gegenüber der adressierten Person zum Ausdruck zu bringen. Von sprachwissenschaftlicher Seite wurde daher auch darauf hingewiesen, dass die Beschimpfung ins "pseudo-assertive" tendiert. ${ }^{54}$ >Volle Missachtungen dagegen besitzen einen sozialen Gehalt, sie nehmen Bezug auf sozial bewertete Eigenschaften, Werte oder Normen (zum Beispiel der Intelligenz, Ordentlichkeit oder Klugheit), die eine Adressatin in einer gegebenen Situation gut oder weniger gut erfüllt. Wenn sich bei einem gemeinsamen Essen unter Freunden jemand ungeschickt benimmt, wäre die spöttische Bemerkung >Du frisst wie ein Schwein eine volle Beleidigung. Die >Fülle dieser Missachtungsform bezieht sich folglich darauf, dass die Adressatin unter Rückbezug auf seine konkrete Verhaltensweise und auf wirkmächtige soziale Normen und Werte abgewertet wird.

52 Eine scheiternde Äußerung ist für Austin eine `Fehlzündung` [misfire]; Austin, Zur Theorie der Sprechakte, a.a.O., S. 38.

53 Bourdieu, Was heißt sprechen?, a.a.O., S. 82.

54 Ermen, Ilse, Fluch - Abwehr - Beschimpfung, Bern u. a. 1996, S. 58, vgl. auch Sornig, »Beschimpfungen«, a.a.O., S. 150-170, hier S. 154. Silver u. a. drücken es so aus: »[...] >mother-fucker is an extremely potent insult. Could this insult be insulting because the insulter, the insulted, or an audience might believe that we actually had enacted the primal scene? Unlikely in the extreme; yet this insult is quite effective. « (Silver, Maury / Rosaria Conte / Maria Miceli / Isabella Poggi, »Humiliation: Feeling, Social Control and the Construction of Identity«, in: Journal for the Theory of Social Behaviour, Heft 3, Bd. 16, 1986, S. 269-283, hier S. 272 f.) 
Zuschreibung und Festschreibung: Missachtungen können, wie Bourdieu sagt, einen Unterschied »aus dem Nichts « hervorbringen oder bereits geschaffene Unterschiede sanktionieren. ${ }^{55}$ Die Zuschreibung >erfindet das adressierte Subjekt neu. In einer Art öffentlichen Anklage zielt sie darauf ab, die aktuelle soziale Position der Adressatin zu zerstören, um eine neue, schlechtere Position zuzuweisen - und zwar dadurch, dass sie einen Dritten oder eine dritte Instanz von der Abwertung der Adressatin, von der sie spricht, überzeugt. Für die Zuschreibung spielt folglich der Dritte in Form des Zeugen eine bedeutende Rolle. Mit Hilfe des Zeugen kann einem behaupteten Unterschied soziale Geltung verschafft werden. Indem der Zeuge >über-zeugt` wird, erlangt der Unterschied nicht nur Bekanntheit, sondern wird auch anerkannt und damit wirkmächtig gemacht. Bei einem spöttischen Witz, z. B. über die scheinbare Verlogenheit des Gegenübers, ist es aus diesem Grund so wichtig, das Publikum auf die eigene Seite zu ziehen - das Lachen des Publikums bestätigt den Erfolg der Zuschreibung. Während Zuschreibungen Unterschiede erst entstehen lassen, machen sich Festschreibungen bestehende Unterschiede zunutze. Dem Menschen mit Behinderung, der als »Krüppel«, der Homosexuellen, die als »Lesbe«, oder die Migrantin, die als »Kanake« angesprochen wird, wird nicht in ein neues soziales Sein zugeschrieben, sondern die Position, die sie im Sozialen einzunehmen gezwungen ist, wird von Neuem als entwertet und minderwertig dargestellt. Die adressierte Person wird nicht neu erfunden, sondern ihre bereits bestehenden Eigenschaften werden aufgegriffen und in wirkmächtige hierarchische Klassifikationen eingeschrieben. Die Wirksamkeit der Festschreibung ist dabei umso größer, je mehr sie den Anschein erwecken kann, dass sie auf >natürlichen` Differenzen, wie Hautfarbe, Alter, Geschlecht beruht.

Graduelle und kategoriale Missachtung: Missachtungen bedienen sich oft zweier unterschiedlicher Klassifikationslogiken: im Anschluss an Foucault möchten wir das kategoriale Regime der Norm und das graduelle Regime der Normalität unterscheiden. ${ }^{56}$ Betrachten wir zunächst das Regime der Norm, das kategorialen Klassifikationen zugrunde liegt, etwas genauer. Die Norm zieht einen Schnitt, sie kennt nur die Zweiwertigkeit von Inklusion und Exklusion. Etwa zwischen den Menschen, die Mitglieder eines Vereins sind, und denen, die es nicht sind, zwischen den Menschen, die eine deutsche Staatsbürgerinnenschaft haben, und denen, die sie nicht haben, oder zwischen den Menschen, die ein körperliches Stigma tragen, und solchen, die keines haben. Die Grenzen, die durch soziale Klassifikationen gezogen werden, können unterschiedlich durchlässig sein, sie reichen von offensichtlich sozialen bis hin zu naturalisierten Unterscheidungen. Mitglied eines Vereins zu werden setzt normalerweise nicht mehr voraus, als einen Antrag zu stellen. Eine Staatsbürgerinnenschaft zu erhalten setzt

55 Bourdieu, Was heißt sprechen?, a.a.O., S. 113.

56 Foucault, Michel, Geschichte der Gouvernementalität I. Sicherheit, Territorium, Bevölkerung. Vorlesungen am Collège de France 1977-1978, hg. von Michel Sennelart, Frankfurt / Main 2004, Vorlesung 3, S. 87-133. 
dagegen eine ganze Reihe von Bedingungen voraus, die nicht für alle Menschen gegeben sind, und ob wir ein körperliches Stigma tragen, liegt normalerweise gar nicht in unserer Hand. Den grundlegend binären Charakter der Norm betont auch Sighard Neckel, wenn er von der »disjunktiven Logik kategorialer Klassifikationen « spricht. ${ }^{57}$ Indem kategoriale Klassifikationen qualitative Urteile der Andersartigkeit fällen, stellen sie ein Nebeneinander wechselseitig sich ausschließender Kategorien her, es gibt keine Schnittmengen oder Überlappungen. Es handelt sich um eine Logik des Entweder-Oder, durch die jenseits aller Gemeinsamkeiten ein grundlegender Unterschied zwischen zwei Gruppen hervorgebracht wird.

Dem graduellen Regime der Normalität, so Foucault, geht es hingegen darum, das Subjekt in seiner individuellen Schicksalhaftigkeit im Vergleich zu anderen zu erfassen. Daher tendiert dieses Regime nicht dazu, einfache binäre Oppositionen aufzustellen, sondern durch die Einbeziehung vieler anderer Schicksale ein hierarchisches Kontinuum zu schaffen, auf dem die Einzelnen verteilt werden. Etwa die >Hochintelligenten<, die >Gescheiten<, die >durchschnittlich Begabten<, die >Förderbedürftigen $\prec$ und die >Hoffnungslosen $\diamond$. Das adressierte Subjekt ist hier nicht einfach >in` oder >out`, sondern auf einem Platz in einem abgestuften Rangsystem verortet. Während das Regime der Norm nur die >Normalen` und die >Anormalen` kennt, existiert für das Regime der Normalität eine Abstufung zwischen vielen einzelnen Fällen. Das graduelle Regime der Normalität situiert ein Subjekt nicht einfach diesseits oder jenseits einer Grenze, sondern es verortet auf einer Linie. Bewertungen werden an dem Maßstab eines Mehr oder Weniger, Größer oder Kleiner gemessen und so in eine kontinuierliche Rangfolge gebracht. Im Gegensatz zu kategorialen Klassifikationen wird so eine Gemeinsamkeit der jeweils bewerteten Merkmale vorausgesetzt. Es handelt sich um eine soziale Logik, die nicht auf Ausschluss, sondern auf Abständen beruht. Auch wenn folglich graduelle Klassifikationen für Missachtungsakte oft ähnlich schmerzhafte Effekte zeitigen können wie kategoriale, bleibt festzuhalten, dass kategoriale Klassifikationen im Allgemeinen tiefgreifendere Effekte hervorrufen können. Das hat seinen Grund darin, dass bei graduellen Klassifikationen zumindest prinzipiell Übergänge zu anderen, überlegenen Positionen möglich oder auch nur denkbar sind. Bei sehr vielen kategorialen Klassifikationen scheint diese Option von vornherein ausgeschlossen, weil die sozialen Grenzziehungen sehr stark naturalisiert wurden und deshalb als etwas Unveränderbares wahrgenommen werden.

Herabwertung und Entwertung: Auch wenn sprachliche Gewalt zugleich Destruktion wie auch Produktion ist, so dominiert in konkreten Akten sprachlicher Gewalt meist eines der beiden Szenarien. Im einen Fall steht der Verlust einer übergeordneten Position im Vordergrund, im anderen der Verweis auf eine unter-

57 Neckel, Sighard / Ferdinand Sutterlüty, »Negative Klassifikationen. Konflikte um die symbolische Ordnung sozialer Ungleichheit«, in: Wilhelm Heitmeyer u.a. (Hg.), Integrationspotenziale einer modernen Gesellschaft, Wiesbaden 2005, S. 409-428. 
geordnete. Bei der Herabwertung dominiert der Aspekt, dass die Adressatin innerhalb eines hierarchischen Schemas ihre Überlegenheit verliert. Sie wird $>$ von ihrem Sockel geholt $<$, wie wir im Alltag sagen. Bei der Entwertung dagegen dominiert der Aspekt der Unterordnung: Die Adressatin wird auf eine minderwertige Position verwiesen. Auf der einen Seite steht also der Verlust einer Überlegenheit, auf der anderen die Produktion einer Unterlegenheit. ${ }^{58}$ Beispiele für Herabwertungen wären das `Duく anstelle eines `Sieく, mit dem ich meinen Vorgesetzten anspreche. Oder die spöttische Bemerkung, die einen Physiker trifft, nachdem er von einem Laien aufgrund eines Fehlers korrigiert wurde. Bei Entwertungen dagegen wird die Adressatin auf einen minderwertigen sozialen Platz verwiesen. Beispiele dafür sind etwa rassistische Äußerungen oder abwertende Äußerungen gegenüber Menschen mit Behinderung. Solche Äußerungen haben eine Unterordnung des Gegenübers zur Folge, sie rücken es an den sozialen Rand. Indem die Adressatin aus dem Kreis der Normalen ausgeschlossen wird, scheint sie durch eine unüberwindliche Kluft von der Sprecherin getrennt zu sein.

Abweisung und Ausschließung: Zu Missachten bedeutet immer auch Abzuweisen. Soziale Prozeduren der Abweisung demonstrieren den Abgewiesenen, dass sie der Nähe nicht wert sind. Eine Person zu meiden, sie nicht anzusprechen, sie zu ignorieren, ihre Äußerungen nicht zu erwidern, zeigt der Betroffenen, dass sie verachtenswert und mangelhaft ist. Dem Subjekt, das wir herabwerten, räumen wir keinen legitimen Platz innerhalb unserer sozialen Strukturen ein. Eine Abweisung geht daher immer auch mit einer Abwertung der Adressatin einher. Diese Verbindung von Abweisung und Abwertung bringen wir oft auch ganz deutlich zum Ausdruck: Etwa in Äußerungen wie »Du Null« oder »Du Niemand $\ll$.

Eine Abweisung kann sich bis zur Ausschließung steigern. Während gewöhnliche Schimpfworte eine Distanzierung nur punktuell und individuell vollziehen, kennzeichnet die Ausschließung eine dauerhafte und kollektive Dimension. Das wird deutlich, wenn man betrachtet, wie im Deutschland der NS-Zeit Juden und Jüdinnen der Zutritt zu Lokalen und Geschäften durch Schilder mit der Aufschrift »Juden und Hunde unerwünscht« verboten wurde. Oder ähnlich im südafrikanischen Apartheidsregime, in dem Parkbänke mit der Aufschrift »Whites only« Schwarzen den Zugang zu bestimmten Orten verwehren sollten. Heinrich Popitz argumentiert in seinem Buch Phänomene der Macht auf ganz ähnliche Weise: Am einen Ende der Missachtungsskala sieht er Praktiken der Abweisung, die die soziale Teilhabe einschränken - wie Distanzierungen, NichtbemerkenWollen oder Kontaktvermeidung. Am anderen Ende stehen Praktiken der Ausschließung, die einen vollkommenen gesellschaftlichen Ausschluß oder eine Ent-

58 Eine Unterscheidung unterschiedlicher Missachtungsformen entlang ähnlicher Demarkationslinien findet sich auch bei Honneth, Axel, Kampf um Anerkennung. Zur moralischen Grammatik sozialer Konflikte, Frankfurt / Main 1994, S. 212-225, und Margalit, Politik der Würde, a.a.O., S. 114-141. 
mündigung vollziehen und zur Isolation des Betroffenen führen. ${ }^{59}$ Die Ausschließung generalisiert also die Abweisung in einer sozialen und einer zeitlichen Hinsicht: In der Ausschließung wird man nicht nur von einer einzelnen Person abgewiesen, sondern von einer Vielheit an Personen. Und man wird nicht nur zu einem bestimmten Moment, sondern in der Dauer der Zeit abgewiesen.

\section{Herausforderung und Erwiderung}

Die verletzende Rede ist kein monologischer, sondern ein kommunikativer Akt. Viele Theorien des Performativen denken den Sprechakt jedoch zumeist allein innerhalb eines monologischen Äußerungsszenarios. Doch schon die Frage des Priesters an das Brautpaar »Wollen sie die Anwesende zur Frau nehmen? « zeigt die latente Dialogizität performativer Akte. Sie können von einer Seite eingeleitet und von der anderen Seite zu Ende gebracht werden - die performative Macht wird dann nicht einseitig ausgeübt, sondern zirkuliert zwischen Sprecherin und Adressatin. Nimmt man dieses Szenario als Ausgangspunkt, dann zeigt sich, dass performative Akte ein Schwellenstadium besitzen. Sie können in der Schwebe sein. Und in diesem Schwellenzustand ist noch völlig ungeklärt, ob und wie der performative Akt seine Kraft entfalten wird; seine endgültige Richtung erhält er erst, wenn er durch eine Antwort vollendet wird.

Jede Ansprache birgt die Möglichkeit einer Antwort, denn sie spricht ihr Gegenüber als soziales Wesen an - als solches ermächtigt, kann dieses immer von der Möglichkeit der Erwiderung Gebrauch machen. Sprachliche Gewalt enthält für die Sprecherin immer die Gefahr, auf eine Antwort zu stoßen. Diese Antwort kann die Gewalt unter Umständen umwenden und eine ermächtigende Form annehmen, etwa wenn eine Beleidigung von der Adressatin in der >gleichen Münze < heimgezahlt wird. ${ }^{60}$ Das hat zur Folge, dass sich das gewaltsame Sprechen oftmals nicht in einem singulären Äußerungsakt erschöpft, sondern in ein >Wortgefecht ausartet, in dem sich Ansprache und Antwort solange abwechseln, bis eine Person >geschlagen ist. Weil der Struktur der Ansprache in einem grundlegenden Sinne die Möglichkeit zur Antwort innewohnt, ist der Ausgang sprachlicher Gewalt daher zunächst einmal immer ungewiss.

Das bedeutet jedoch nicht, dass sich Rede und Gegenrede immer symmetrisch gegenüberstehen würden, denn jede verletzende Äußerung findet in einem Machtkontinuum statt, indem sich die Rede mit unterschiedlicher Kraft zu sättigen vermag. Es kann daher ein Ungleichgewicht im Kräfteverhältnisses zwischen Sprecherin und Hörerin existieren, durch welches die Möglichkeit des Antwortens vorstrukturiert ist. Das zeigt sich in dem Handlungsspielraum, der für die Antwort zur Verfügung steht. Ein Beispiel: Die Migrantin, die rassistisch beleidigt wurde, kann in der konkreten Situation auf die rassistische Äußerung nicht selbst mit einer rassistischen Äußerung antworten. Sie mag die weiße deutsche

59 Popitz, Phänomene der Macht, a.a.O., S. 45.

60 Freud, Der Witz und seine Beziehung zum Unbewussten, a.a.O., S. 83. 
Sprecherin vielleicht als »Streberdeutsche« zu beleidigen versuchen, aber vermutlich wird diese Äußerung scheitern. Die Bedeutung und die Rolle der Äußerung ist klar - auch ihre herabwertende Intention -, aber ihr fehlt jede Kraft. Je stärker die Adressatin der Sprecherin unterlegen ist, desto weniger Möglichkeiten des Zurücksprechens bleiben ihr. Auch wenn die Möglichkeit zur Antwort daher jeder Ansprache ihrer Struktur nach eingeschrieben ist, kann sich diese Möglichkeit letztlich als die Unmöglichkeit einer wirkungsvollen Antwort entpuppen. Der Grund dafür liegt darin, dass die Angesprochenen oftmals nicht in der Lage sind, wirkmächtig mit der Stimme eines Dritten zu sprechen. Von der Möglichkeit zur Antwort Gebrauch zu machen, muss nicht immer heißen, erfolgreich von ihr Gebrauch zu machen - die Antwort kann auch wirkungslos bleiben.

Eine Antwort kann jedoch nicht nur wirkungslos sein, sie kann auch verunmöglicht werden. Und zwar durch die Ansprache selbst, die Strategien einsetzen kann, die Möglichkeit der Antwort ganz außer Kraft zu setzen. Eine Möglichkeit, die Antwort zu unterbinden, liegt im Einsatz effektvoller Rhetoriken. Durch sie soll die Möglichkeit zur Antwort bereits in der Ansprache vorauseilend genommen werden. Subtile sprachliche Mechanismen wie der Witz, die Anspielung oder die Ironie können dazu dienen, den missachtenden Gehalt auf einer konnotativen Ebene zum Ausdruck zu bringen. Der beleidigende Gehalt kann hintergründig in die Worte eingestreut werden. Das hat den Vorteil, dass nicht klar ist, ob die Sprecherin überhaupt etwas gesagt hat bzw. sie abstreiten kann, dass sie es ssor gemeint hat. Die Antwort geht in diesem Fall ins Leere, sie findet keinen Halt. Eine andere rhetorische Möglichkeit, einer ermächtigenden Antwort zu entgehen, kann in der lautstarken Beschimpfung bestehen. Das Herausplatzen mit einem Schimpfwort etwa kann dazu dienen, das Gegenüber zu überrumpeln und in einem Zug zu bezwingen. Der Schock soll in den Gliedern sitzen, damit die Möglichkeit, sich zu fangen, genommen ist. Die Adressatin bleibt sprachlos. Die Antwort bleibt aus. Das macht deutlich: Es gibt zwar immer die Möglichkeit, auf verletzende Worte zu Antworten, und diese Antwort kann auch erfolgreich ausfallen, der Spielraum für eine Umwendung sprachlicher Gewalt kann im Konkreten jedoch bis auf ein Minimum reduziert sein.

Gibt es die Möglichkeit zur Antwort, dann kann diese Möglichkeit in einen Zwang zur Erwiderung umschlagen. Bourdieu hat in seinen Studien zur Kabylei herausgearbeitet, dass es sich dabei vor allem um ein Männlichkeitsritual handelt. ${ }^{61}$ Vom Adressaten wird erwartet, dass er seine befleckte Ehre verteidigt. Tut er das nicht, so kann sich die zugefügte Gewalt noch steigern. Der Angesprochene wurde dann nicht nur durch die Worte gedemütigt, sondern zugleich noch als jemand, der nicht auf die Ansprache antworten kann. Die unterlassene Erwiderung des Angesprochenen droht diesen als >feige zu diskreditieren. In der Perspektive des Zwangs zur Erwiderung geht es folglich vor allem darum, dass der

61 Vgl. dazu Bourdieu, Pierre, »Zur Dialektik von Herausforderung und Erwiderung der Herausforderung «, in diesem Band, S. 89-106. 
Adressat zeigt, dass er sich nicht die Stimme rauben lässt. Zu diesem Zweck ist es nicht unbedingt notwendig, eine effektvolle oder ermächtigende Erwiderung hervorzubringen, sondern nur, die Herausforderung überhaupt zu erwidern. Tatsächlich genügt oft schon das bloße Hervorbringen irgendeiner Antwort - auch wenn diese nicht >schlagend ist. Durch die Tatsache, die Ansprache erwidert zu haben, schreibt sich der Angesprochene in den Kreis derjenigen ein, die das Spiel von Herausforderung und Erwiderung wert - und fähig - sind zu spielen. Für viele Kulturen und Subkulturen sind das vor allem Männer. Die Beleidigung dient hier der Herausforderung der Männlichkeit des Gegenübers. Indem der Adressat zurückspricht beweist dieser nicht nur, dass er die Fähigkeit zur Erwiderung besitzt, sondern zugleich auch, dass er sich nicht in einem passivem Schweigen ergibt. Die Erwiderung zeigt, dass der Adressat am maskulinen Kreislauf der Kommunikation teilnehmen und >seinen Mann stehen ২ kann. Die Erwiderung wird hier zum Zwang, denn die Unfähigkeit zur Erwiderung bedeutet hier nicht nur die Beleidigung hinzunehmen, sondern zugleich auch, in der eigenen Männlichkeit in Frage gestellt zu werden.

Sprachliche Gewalt als soziale Praxis zu verstehen, bedeutet die kommunikative Seite des Äußerungsszenarios mit einzubeziehen. Diese Perspektive kann sich jedoch nicht darin erschöpfen, die Möglichkeit der Antwort als Weg zur Umwendung der Gewalt zu erfassen, vielmehr muss auch die Möglichkeit des Antwortens selbst nach ihren Möglichkeitsbedingungen befragt werden. Nach der Antwort zu fragen heißt, auch danach zu fragen, in welchem Rahmen überhaupt eine Antwort gegeben werden kann und unter welchen Umständen eine Antwort sogar zum Zwang wird - erzwungen durch die drohende Gefahr der zusätzlichen Demütigung im Fall einer Unfähigkeit zu antworten.

\section{Literatur}

Althusser, Louis, »Ideologie und ideologische Staatsapparate«, in: ders., Ideologie und ideologische Staatsapparate. Aufsätze zur marxistischen Theorie, Hamburg 1977, S. 108-153.

Apel, Karl-Otto, Diskurs und Verantwortung. Das Problem des Übergangs zur postkonventionellen Moral, Frankfurt / Main 1990.

Austin, John L., Zur Theorie der Sprechakte (How to Do Things With Words), 2. Aufl., Stuttgart, 1979 (engl. Original 1962).

Benedict, Ruth, Chrysantheme und Schwert, Frankfurt / Main 2006.

Benveniste, Emile, »Die Natur der Pronomen«, in: ders., Probleme der allgemeinen Sprachwissenschaft, München 1974, S. 279-286.

— »Über die Subjektivität in der Sprache«, in: ders., Probleme der allgemeinen Sprachwissenschaft, München 1974, S. 287-297.

Bourdieu, Pierre, Meditationen. Zur Kritik der scholastischen Vernunft, Frankfurt / Main 2001. 
- Was heißt sprechen? Zur Ökonomie des sprachlichen Tausches, 2., erw. und überarb. Aufl., 2005.

— »Zur Dialektik von Herausforderung und Erwiderung der Herausforderung«, in diesem Band, S. 89-106.

Buber, Martin, Das dialogische Prinzip, Heidelberg 1984.

Butler, Judith, Hass spricht. Zur Politik des Performativen, Berlin 1998.

- Psyche der Macht. Das Subjekt der Unterwerfung, Frankfurt / Main 2001.

Ermen, Ilse, Fluch - Abwehr - Beschimpfung, Bern u. a. 1996.

Freud, Sigmund, Der Witz und seine Beziehung zum Unbewussten (1905), in: ders., Der Witz und seine Beziehung zum Unbewussten. Der Humor, 6. Aufl., Frankfurt / Main 2001.

Foucault, Michel, Geschichte der Gouvernementalität I. Sicherheit, Territorium, Bevölkerung. Vorlesungen am Collège de France 1977-1978, hg. von Michel Sennelart, Frankfurt / Main 2004.

Garfinkel, Harold, »Bedingungen für den Erfolg von Degradierungszeremonien«, in diesem Band, S. 49-57 (engl. Original 1956).

Gehring, Petra, »Die Wiederholungs-Stimme. Über die Strafe der Echo«, in: Doris Kolesch / Sybille Krämer (Hg.), Stimme, Frankfurt / Main 2006.

Graumann, Carl F., »Verbal Discrimination: A Neglected Chapter in the Social Psychology of Aggression «, in: Journal for the Theory of Social Behaviour, Heft 1, Bd. 28, 1998, S. 41-61.

Habermas, Jürgen, Theorie des kommunikativen Handelns, 2 Bde., Frankfurt / Main 1995.

Hegel, Georg Wilhelm Friedrich, Phänomenologie des Geistes (1807), in: ders., Werke 3, Frankfurt / Main 1986.

Honneth, Axel, Kampf um Anerkennung. Zur moralischen Grammatik sozialer Konflikte, Frankfurt / Main 1994.

— »Unsichtbarkeit. Über die moralische Epistemologie von >Anerkennung «", in: ders., Unsichtbarkeit. Stationen einer Theorie der Intersubjektivität, Frankfurt / Main 2003, S. 10-27.

Imbusch, Peter, »Der Gewaltbegriff«, in: Wilhelm Heitmeyer / John Hagan (Hg.), Internationales Handbuch der Gewaltforschung, Wiesbaden 2002, S. 26-57.

Kojève, Alexandre, Hegel. Kommentar zur Phänomenologie des Geistes. Mit einem Anhang: Hegel, Marx und das Christentum, Frankfurt / Main 1975 (franz. Original 1947).

Kripke, Saul A., Name und Notwendigkeit, Frankfurt / Main 1981.

Lacan, Jacques, »Funktion und Feld des Sprechens und der Sprache in der Psychoanalyse«, in: ders., Schriften I, Olten 1973, S. 71-169.

Langton, Rae, »Sprechakte und unsprechbare Akte«, in diesem Band, S. 107146.

Levinas, Emmanuel, Ethik und Unendliches. Gespräche mit Philippe Nemo, hg. v. Peter Engelmann, Wien 1996. 
- Jenseits des Seins oder anders als Sein geschieht, Freiburg, München 1998.

- Humanismus des anderen Menschen, Hamburg 2005.

Liebsch, Burkhard, »)Sprechende〈 Gewalt«, in: Kristin Platt (Hg.), Reden von Gewalt, München 2002, S. 150-174.

Malinowski, Bronislaw, »Das Problem der Bedeutung in primitiven Sprachen «, in: Charles K. Ogden / Ivor A. Richards, Die Bedeutung der Bedeutung, Frankfurt / Main 1974, S. 323-384 (engl. Original 1923).

Margalit, Avishai, Politik der Würde. Über Achtung und Verachtung, Frankfurt / Main 1999.

Neckel, Sighard / Ferdinand Sutterlüty, »Negative Klassifikationen. Konflikte um die symbolische Ordnung sozialer Ungleichheit«, in: Wilhelm Heitmeyer u. a. (Hg.), Integrationspotenziale einer modernen Gesellschaft, Wiesbaden 2005, S. 409-428.

Nunner-Winkler, Gertrud, »Mobbing und Gewalt in der Schule. Sprechakttheoretische Überlegungen«, in: Westend, Heft 1, 2004, S. 91-100.

Patterson, Orlando, Slavery and Social Death. A Comparative Study, Cambridge u. a. 1982.

Popitz, Heinrich, Phänomene der Macht, 2., stark erw. Aufl., Tübingen 1992.

Ricœur, Paul, Das Selbst als ein Anderer, München 1996.

Rorty, Richard, Kontingenz, Ironie und Solidarität, Frankfurt / Main 1992.

Scarry, Elaine, Der Körper im Schmerz. Die Chiffren der Verletzlichkeit und die Erfindung der Kultur, Frankfurt / Main 1992.

Siep, Ludwig, Anerkennung als Prinzip der praktischen Philosophie. Untersuchungen zu Hegels Jenaer Philosophie des Geistes, Freiburg u. a. 1979.

Silver, Maury / Rosaria Conte / Maria Miceli / Isabella Poggi, »Humiliation: Feeling, Social Control and the Construction of Identity«, in: Journal for the Theory of Social Behaviour, Heft 3, Bd. 16, 1986, S. 269-283.

Sornig, Karl, »Beschimpfungen«, in: Grazer Linguistische Studien. Sprache \& Gesellschaft, Heft 1, 1975, S. 150-170.

Till, Sabine, Sprache und Stimme bei Lacan und Levinas, Magistraarbeit, Institut für Philosophie, Freie Universität Berlin 2005.

Todorov, Tzvetan, Abenteuer des Zusammenlebens. Versuch einer allgemeinen Anthropologie, Frankfurt / Main 1998.

Trotha, Trutz von, »Zur Soziologie der Gewalt«, in: ders. (Hg.), Soziologie der Gewalt, Sonderheft Kölner Zeitschrift für Soziologie und Sozialpsychologie, Wiesbaden 1997, S. 9-56.

Tugendhat, Ernst, Selbstbewusstsein und Selbstbestimmung. Sprachanalytische Interpretationen (1979), 6. Aufl., Frankfurt / Main 1997.

Watzlawik, Paul / Janet H. Beavin / Don D. Jackson, Menschliche Kommunikation. Formen, Störungen, Paradoxien, 10. Aufl., Bern u. a. 2000.

Wittgenstein, Ludwig, Das blaue Buch, 9. Aufl., Frankfurt / Main 2000. 


\section{Inhalt}

Steffen Kitty Herrmann und Hannes Kuch

Verletzende Worte. Eine Einleitung

Sybille Krämer

Sprache als Gewalt oder: Warum verletzen Worte?

Harold Garfinkel

Bedingungen für den Erfolg von Degradierungszeremonien

Penelope Brown und Stephen C. Levinson

Gesichtsbedrohende Akte

Pierre Bourdieu

Die Dialektik von Herausforderung und Erwiderung

der Herausforderung

Rae Langton

Sprechakte und unsprechbare Akte

Carl Friedrich Graumann und Margret Wintermantel

Diskriminierende Sprechakte. Ein funktionaler Ansatz

Hannes Kuch und Steffen Kitty Herrmann

Symbolische Verletzbarkeit und sprachliche Gewalt

Petra Gehring

Über die Körperkraft von Sprache 
Pascal Delhom

Die geraubte Stimme

Burkhard Liebsch

Nach dem angeblichen Ende der ıSprachvergessenheit «: Vorläufige

Fragen zur Verletzung Anderer in und mit Worten

Stefan Deines

Verletzende Anerkennung. Über das Verhältnis von Anerkennung, Subjektkonstitution und >sozialer Gewalt

Thomas Markert

Zur Praxis verbaler Gewalt unter Schülerinnen und Schülern

Sonja Kleinke

Sprachliche Strategien verbaler Ablehnung in öffentlichen

Diskussionsforen im Internet

Mechthild Hetzel und Andreas Hetzel

Zur Sprache der Sprachlosen. Ebenen der Gewalt in der diskursiven

Produktion von Behinderung

Daniel Loick

Words like violence. Konstellationen des Unvernehmens

Die Autorinnen und Autoren

Nachweise 